\title{
Genetic Variability of Eggplant Germplasm Evaluated under Open Field and Glasshouse Cropping Conditions
}

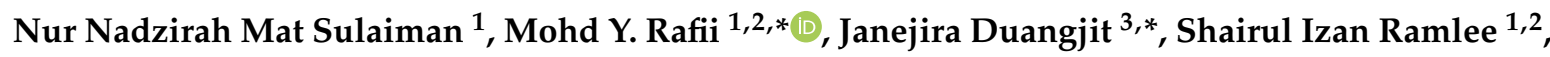 \\ Chalermpol Phumichai ${ }^{4}$, Yusuff Oladosu ${ }^{1}{ }^{(\mathbb{D}}$, Debi Rani Datta ${ }^{1}$ and Ibrahim Musa ${ }^{1}$ \\ 1 Laboratory of Climate-Smart Food Crop Production, Institute of Tropical Agriculture and Food Security, \\ Universiti Putra Malaysia (UPM), Serdang 43400, Selangor, Malaysia \\ 2 Department of Crop Science, Faculty of Agriculture, University Putra Malaysia, Serdang 43400 UPM, \\ Selangor, Malaysia \\ 3 Department of Horticulture, Faculty of Agriculture, Kasetsart University, 50 Ngam Wong Wan Road Lat Yao, \\ Chatuchak, Bangkok 10900, Thailand \\ 4 Department of Agronomy, Faculty of Agriculture, Kasetsart University, 50 Ngam Wong Wan Road Lat Yao, \\ Chatuchak, Bangkok 10900, Thailand \\ * Correspondence: mrafii@upm.edu.my (M.Y.R.); janejira.d@ku.th (J.D.); Tel.: +603-9769-1043 (M.Y.R.); \\ +662-579-0308 (J.D.)
}

Received: 19 February 2020; Accepted: 19 March 2020; Published: 23 March 2020

\begin{abstract}
Knowledge of agro-morphological genetic variation and cropping conditions on vegetative and yield-related traits plays a significant role in varietal improvement and production of eggplant (Solanum melongena L.). Following this premise, the current study was conducted to critically asses the genetic variation of 29 eggplant accessions by using agro-morphological characterization evaluated under two cropping conditions, namely, glasshouse and open field. The experiments were laid out in randomized complete block design (RCBD) with three replications. Data on vegetative and yield characteristics were collected and subjected to analysis of variance (ANOVA) using SAS 9.4, while variance components were estimated manually. The results obtained from the analysis of variance indicated a highly significant difference $(p \leq 0.01)$ for all characteristics studied in both cropping conditions. The evaluated accessions were grouped into six major clusters based on agro-morphological traits using Unweighted Pair Group Method with Arithmetic mean (UPGMA) dendrogram. Hence, crosses between group I with VI or V could be used to attain higher heterosis and vigor among the accessions. Also, this evaluation could be used as a selection criterion for important yield agronomic traits in eggplant. The methodology and the approaches used may provide a model for the enhancement of other vegetable crop diversity towards adaptability to the cropping condition decision. This result displayed importance for preserving eggplant germplasm for future varietal development and revealed that open field cropping condition is more suitable under Malaysia's agroecology.
\end{abstract}

Keywords: genetic variation; eggplant; cropping condition; yield; agro-morphological characterization

\section{Introduction}

Eggplant (Solanum melongena L.) is one of the important vegetables belonging to the family Solanaceae, which comprises other significant crop species including chilli (Capsicum annuum L.), tomato (Solanum lycopersicum L.), tobacco (Nicotiana tabacum L.), and potato (Solanum tuberosum L.). Eggplant is an old world crop species, unlike other members of the Solanaceae family [1]. According to [1], eggplant's ancestor, Solanum incanum or eggplant bitter apple, pre-domesticated in the subtropical species and is a native of West Asia and North Africa that is used as the source of resistance to 
drought and variation for phenolics content in eggplant breeding programs [2]. The global eggplant production statistic in 2017 was 52.31 million tons [3], going up by 2.18\% against the previous year. This global eggplant production peaked in 2017, and the growth trend pattern is likely to be on a continuous increase [3]. China and India are the top eggplant producing countries in the world followed by Egypt, Turkey, Indonesia, Iran, Philippines, Spain, Mexico, Japan, Italy, and Syria (http://www.fao.org/faostat/en/\#data/QC). In the context of Malaysia, vegetable production-especially eggplant-has been low with only 39,311.5 metric tons [4], and one factor identified was due to lack of genetic resources. This has forced the country to rely on importation of vegetable seeds to meet 265 tons annually, but locally they can only meet 13 tons [5], leading to a deficit of 252 tons/year, which is being imported from overseas [6]. With limited eggplant cultivation and studies, there is a duty call for awareness of the importance of utilization and development of our available varieties gearing towards food security and the high-value vegetable market of Malaysia. The local production could improve through the exploration of germplasm. The existence of compelling high genetic variation is critical for expanding the stricken eggplant genetic base and advancing current germplasm, whether it is local or commercial germplasm.

Generally, morphological characterization is the first step in exploring eggplant genetic variation. Hence, there is a wide variation in eggplant habitats as well as vegetative and agro-morphological characteristics [7]. Besides, the evaluation of genetic variation and adaptation to climatic conditions using agronomic traits has been the focus of research in the last decade [8]. Recently, taste, texture, and appearance are among the considered factors alongside nutrient compositions [9] that are being emphasized to meet consumer demand. In conjunction with that, eggplant has been bred for improved fruit quality, fruit yield, disease resistance, and adaptation with stable, high yielding performance across heterogeneous growing areas. Eventually, this marker was found interesting and was best applied by plant breeders due to easy scoring, low cost, and rapid method and evergreen evaluations. Also, investigation of these qualities required non-complex tools and equipment, and it could be acquired without explicit biochemical or molecular methods. Specifically, it could be aided with competent multivariate tools such as principal component analysis (PCA) [10], clustering, and discriminate analysis for assessing the genetic diversity of germplasm introduced in heterogeneous crops [11]. In addition, clustering analysis [12] was utilized together with pattern analysis for grouping prior scattered materials in which a collective use of methods was ordinated and classified for investigating the structure of the fundamental basis among germplasm [13]. We need a precise and practical classification of the eggplant germplasm genetic pool in different cultivar groups, which is vital to promoting their use of crop improvement.

A priori, eggplant is one of the model crops that can be grown in a heterogenous macro-environment [14]. However, knowledge of suitable cropping conditions and plant conditions is a prerequisite for cost-effective production. In any geographical area, the prevalent cropping condition is the aggregate results of the previous decisions by individuals, communities, or governments and their agencies. Hence, crop adaptability of the growing conditions such as raining seasons, species, and land use efficiency together with plant growth resources such as irrigation, climate, tradition, and experiences are among the determinant factors for efficient production. In the context of Malaysia, conventional open fields and glasshouses are the two most widely used cropping conditions for eggplant cultivation. None of the studies focused on eggplant genetic variation to simultaneously compare yield performance between these cropping conditions. Therefore, this study was conducted to evaluate genetic variation and establish relationships between vegetative, yield, and yield components using agro-morphological characterization among 29 eggplant accessions from Malaysia, Thailand, and China under two cropping conditions, namely, a fertigation system in the glasshouse and an open field condition. 


\section{Materials and Methods}

\subsection{Planting Materials and Agronomic Practice}

Twenty-nine eggplants accessions, which form three main populations from Malaysia, Thailand, and China were used in this study, as presented in Table 1. The evaluation was conducted at Field 10 (S8) at Universiti Putra Malaysia (UPM), Serdang, Selangor, Malaysia, which is geographically located between $2^{\circ} 59^{\prime}$ north latitude and $101^{\circ} 42^{\prime}$ east longitude, with $45 \mathrm{~m}$ above sea level altitude. The other experiment was conducted in Fertigation Unit, Ladang 15, Universiti Putra Malaysia (UPM), Serdang, Selangor, Malaysia, which is between $2^{\circ} 59^{\prime}$ north latitude and $101^{\circ} 43^{\prime}$ east longitude, with an altitude of $55 \mathrm{~m}$. The experiment was laid out in randomized complete block design (RCBD) with three replications. The environment is hot humid tropics with high humidity and adequate rainfall throughout the year. The seeds were sown in 104-holes seed germinating trays with 1-2 seeds per cell on peat moss growing medium. After 27 days of sowing, the seedlings were transplanted to mixed soil and peat moss with a ratio of 2:1 in polybags for the hardening phase for another 25 days before being transferred to the field and the glasshouse conditions. In each replication, the eggplant was planted with $50 \mathrm{~cm}$ spacing between the five plants of each accession and $1 \mathrm{~m}$ between the rows. Following the standard cultural practices, agronomic routines and plant maintenance such as fertilizer application, pest and disease management, and weeding were carried out. On a daily basis for the glasshouse fertigation system, the plants were supplied with modified copper formulation fertilizer consisting of $\mathrm{N}\left(200 \mathrm{mg} \mathrm{L}^{-1}\right), \mathrm{P}\left(60 \mathrm{mg} \mathrm{L}^{-1}\right), \mathrm{K}\left(300 \mathrm{mg} \mathrm{L}^{-1}\right), \mathrm{Ca}\left(170 \mathrm{mg} \mathrm{L}^{-1}\right), \mathrm{Mg}\left(50 \mathrm{mg} \mathrm{L}^{-1}\right), \mathrm{Fe}\left(12 \mathrm{mg} \mathrm{L}^{-1}\right)$, $\mathrm{Mn}\left(2 \mathrm{mg} \mathrm{L}^{-1}\right), \mathrm{B}\left(1.5 \mathrm{mg} \mathrm{L}^{-1}\right), \mathrm{Zn}\left(0.1 \mathrm{mg} \mathrm{L}^{-1}\right), \mathrm{Cu}\left(0.1 \mathrm{mg} \mathrm{L}^{-1}\right)$, and Mo $\left(0.2 \mathrm{mg} \mathrm{L}^{-1}\right)$ [15] while the electron conductivity (EC) reading increased in succession according to the growing phase (0.5-3.0). In the aspect of pest and disease mitigation strategies, several pesticides were applied as recommended by the Department of Agriculture, Malaysia (http://jpn.penang.gov.my/index.php/perkhidmatan/ teknologi-tanaman/sayur-sayuran/78-terung-sp-3424).

Table 1. List of 29 eggplants accessions.

\begin{tabular}{|c|c|c|c|c|c|}
\hline No. & Accessions Code & Accessions Name & Origin Country & Fruit Type & Collection Source \\
\hline 2 & $2 \mathrm{TL}$ & CT Long Green Eggplant 01166 & Thailand & Long & Village Market \\
\hline 4 & $4 \mathrm{TR}$ & CT El Ryu Eggplant 636 & Thailand & Round & Village Market \\
\hline 5 & 5TR & MOP Eggplant 548 & Thailand & Round & Village Market \\
\hline 6 & $6 \mathrm{TL}$ & MOP Eggplant 969 & Thailand & Long & Village Market \\
\hline 9 & 9ML & Eggplant B. VE-023 $\mathrm{F}_{1}$ Hybrid Long & Malaysia & Long & Commercial market \\
\hline 10 & $10 \mathrm{ML}$ & White Shining Eggplant $330 \mathrm{~F}_{1}$ Hybrid & Malaysia & Long & Commercial market \\
\hline 11 & $13 \mathrm{MR}$ & Round Eggplant MTe2 & Malaysia & Round & MARDI Gene Bank \\
\hline 12 & $14 \mathrm{ML}$ & Terung Belacan D/No 04-1272 & Malaysia & Long & MARDI Gene Bank \\
\hline 13 & $15 \mathrm{ML}$ & Terung Rapuh Unggu 76 & Malaysia & Round & MARDI Gene Bank \\
\hline 14 & $16 \mathrm{ML}$ & L. Little Nyonya 313 & Malaysia & Long & Commercial market \\
\hline 19 & $21 \mathrm{ML}$ & MChina-3 & China & Round & Commercial market \\
\hline 20 & $22 \mathrm{ML}$ & Mukta Keshi & Malaysia & Long & Commercial market \\
\hline 21 & $23 \mathrm{ML}$ & Makra Begun & Malaysia & Round & Commercial market \\
\hline 22 & $25 \mathrm{ML}$ & Brinjal Bhagan & Malaysia & Long & Commercial market \\
\hline 23 & $26 C L$ & China 1 & China & Long & Commercial market \\
\hline 24 & $27 \mathrm{CL}$ & TESH Eggplant 204 & Malaysia & Long & Commercial market \\
\hline 25 & $29 \mathrm{MN}$ & NTH 08-0031 & Malaysia & Long & MARDI Gene Bank \\
\hline 26 & $30 \mathrm{MN}$ & NTH 08-0077 & Malaysia & Round & MARDI Gene Bank \\
\hline 27 & $32 \mathrm{MN}$ & NTH 08-0131 & Malaysia & Long & MARDI Gene Bank \\
\hline 28 & $34 \mathrm{CL}$ & China 3 & China & Long & Commercial market \\
\hline 29 & $35 \mathrm{CL}$ & China 2 & China & Long & Commercial market \\
\hline
\end{tabular}

Note: MARDI = Malaysian Agricultural Research \& Development Institute, TR = Thailand Round, TL = Thailand Long, $\mathrm{ML}=$ Malaysian Long, $\mathrm{MR}=$ Malaysian Round, $\mathrm{MN}=$ Malaysian Native, $\mathrm{CL}=$ China Long. 


\subsection{Data Collection}

Thirteen sets of agro-morphological data were collected and measured from the two planting conditions following the description of the International Boards for Plant Genetic Resources [16] and European Eggplant Genetic Resources Network [17]. To obtain the means of the variables in each plot, five fruits were chosen at random from each of the three tagged plants in the middle of each replication during the harvest. The harvest was carried out five times subject to the productivity of plants that might vary between the accessions. Harvesting frequency and respective number of fruits taken were recorded (data not shown). Plants were evaluated on the yield components fruit girth (FGI), diameter of fruit (DFR), fruit length (FLE), fruit length to width ratio (FLW), total number of fruit (TNF), number of fruit per bunch (NFB), average fruit weight (AFW), and fruit yield per plant (FYP). These also included vegetative parameters such as number of primary branches (NPB), plant height (PHE), stem diameter (SDM), plant spread (PSP), and days to first flowering (DFF). All data measurement and observations were accomplished on the same day to reduce variation in plant growth developmental stage or environmental changes.

\subsection{Statistical Analysis}

All vegetative, yield, and yield-related data in collection were subjected to analysis of variance (ANOVA) using the SAS version 9.4 (SAS Institute Inc., Cary, NC, USA), while means comparisons were separated with least significant difference (LSD) at $5 \%$ level of significance. Additionally, grand mean, standard deviation, and coefficient of variation (CV) were recorded for each trait measured. Together with the analysis of variance (ANOVA), nested design analysis was a hierarchical design plotted with fruit type and the whole set of attributes of eggplant as an interest of evaluation in eggplant accession. Among the 29 accessions subjected in this agro-morphological analysis, ten accessions were the round type of eggplant, and 19 accessions were long shape type. Both long and round fruit types of specifically assigned eggplants were subsampled under accession and eventually made the fruit type within accession. Accession has a higher level as compared to fruit type.

Genetic relationships among the eggplant germplasm were determined using the unweighted pair group method with arithmetic mean (UPGMA algorithm) and sequential agglomerative hierarchical non-overlapping (SAHN) methods. Cluster trees [18] are important multivariate tools to assess genetic variation among the eggplant's germplasm under two cropping conditions. Utilizing the basis of comparable vegetative and yield components traits, the grouping of individual accessions was exposed by cluster analysis according to similarity and relatedness of eggplants. Other than that, restricted maximum likelihood (REML) using PROC VARCOMP in SAS version 9.4 (SAS Institute Inc., Cary, NC, USA) was used for variance components estimations. Genetic parameters such as genotypic coefficient variance (GCV), phenotypic coefficient variance (PCV), heritability in the broad sense $\left(\mathrm{H}^{2}{ }_{\mathrm{B}}\right)$, and genetic advance (GA) were calculated using the following equation [19].

$$
\begin{gathered}
\mathbf{P C V}=\frac{\sqrt{\sigma_{\mathrm{P}}^{2}}}{\overline{\mathrm{X}}} \times 100 \\
\mathrm{GCV}=\frac{\sqrt{\sigma_{\mathrm{\sigma}}^{2}}}{\overline{\mathrm{X}}} \times 100
\end{gathered}
$$

where $\sigma_{\mathrm{P}}^{2}$ is the phenotypic variance, $\sigma_{\mathrm{g}}^{2}$ is the genotypic variance, and $\overline{\mathrm{X}}$ is the traits mean. PCV and GCV were classified as low $(<10 \%)$, moderate $(10-20 \%)$, and high $(>20 \%)$, as described by [20]. 
Percentage of broad-sense heritability was estimated as the proportion of phenotypic variance $\left(\sigma_{\mathrm{P}}^{2}\right)$ to genotypic variance $\left(\sigma_{\mathrm{g}}^{2}\right)$ as indicated below:

$$
h_{B}^{2}=\frac{\sigma_{\mathrm{g}}^{2}}{\sigma_{\mathrm{P}}^{2}} \times 100
$$

where $\sigma_{\mathrm{P}}^{2}$ is phenotypic variance, and $\sigma_{\mathrm{g}}^{2}$ is the genotypic variance. Heritability values were estimated and standardized as low $(0-30 \%)$, moderate $(30-60 \%)$, and high $(>60 \%)$ in accordance with Robinson et al. and Assefa et al. [21,22].

Expected genetic advance (GA) percentage was estimated following the method described by Johnson et al. [23].

$$
\mathbf{G A} \%=\mathrm{K} \times \frac{\sqrt{\sigma_{\mathrm{P}}^{2}}}{\overline{\mathrm{X}}} \times h_{B}^{2} \times 100
$$

where $\mathrm{K}$ is a constant that represents the selection intensity. At the value when $\mathrm{k}$ is at $5 \%$, the rate is 2.06. $\frac{\sqrt{\sigma_{P}^{2}}}{\bar{x}}$ is the phenotypic standard deviation, and $h_{B}^{2}$ is the broad-sense heritability value. GA values of $0-10 \%, 10-20 \%$, and $>20 \%$ are low, intermediate, and high, respectively $[21,24]$.

\section{Results and Discussion}

\subsection{Yield and Yield Components Across Two Cropping Conditions}

The pooled analyses of variance for yield and yield traits from two cropping conditions are presented in Table 2. Highly significant differences $(p \leq 0.01)$ were observed among the accessions and the fruit types within the accessions for all yield and yield parameters measured. Similarly, highly significant differences $(p \leq 0.01)$ were recorded in the cropping conditions for all yield and yield-related traits except for the number of fruits per bunch (NFB). Next, highly significant differences $(p \leq 0.01)$ were observed in fruit type for all the yield and the yield-related parameters except for average fruit weight (AFW) and fruit yield per plant (FYP), where non-significant differences were observed. On the other hand, the interaction between cropping conditions with the accession showed highly significant differences $(p \leq 0.01)$ for the most important yield and yield component traits, which were fruit yield per plant (FYP), fruit length (FLE), total number of fruit (TNF), and average fruit weight (AFW). This strongly signified that cropping conditions and agronomic practices play pivotal roles in affecting eggplant varietal aspects in terms of yield and yield components. The result also revealed that there was no sign of replication effect within the cropping condition except for total number of fruits (TNF), average fruit weight (AFW), and fruit yield per plant (FYP). A high coefficient of variation $(\mathrm{CV})$ of more than $40 \%$ was applied to average fruit weight (AFW) and fruit yield per plant (FYP), indicating they were the most diverse quantitative agro-morphological characteristics observed in Table 2. In general, all accessions were strongly varied from each other in terms of yield characteristics. The reason for the significant difference is apparently the differences in their origins that cause the existence of variation in a population [25]. Similarly, several studies have been carried out on phenotypic variation among eggplant accessions. The outcome of this research is in agreement with the findings of Caguiat and Hautea [26]. Hence, this strongly supported a postulation by Naujeer [27] that enhanced yield and improved fruit quality are defined as the main objectives in eggplant breeding program. 
Table 2. Mean square of yield and yield component of 29 eggplant accessions.

\begin{tabular}{|c|c|c|c|c|c|c|c|c|c|}
\hline Sources of Variation & DF & FGI & DFR & FLE & FLW & TNF & NFB & AFW & FYP \\
\hline Cropping condition (C) & 1 & $123.51 * *$ & $55.48 * *$ & $483.34 * *$ & $6.08^{* *}$ & $28,678.25^{* *}$ & $0.02 \mathrm{~ns}$ & $10,836,424.27 * *$ & $295,844,792$ ** \\
\hline Replications within C (R/C) & 4 & $5.24 \mathrm{~ns}$ & $0.81 \mathrm{~ns}$ & $6.14 \mathrm{~ns}$ & $0.20 \mathrm{~ns}$ & $107.24^{* *}$ & $0.05 \mathrm{~ns}$ & $143,806.97^{* *}$ & $3,823,329.5^{* *}$ \\
\hline Accessions (A) & 28 & $95.28 * *$ & $12.37 * *$ & $137.69 * *$ & $10.88^{* *}$ & $1017.08^{* *}$ & $0.59 * *$ & $261,459.39 * *$ & $3,065,055.9 * *$ \\
\hline Fruit types (F) & $(1)$ & $259.02 * *$ & $23.52 * *$ & $2613.71^{* *}$ & $230.36^{* *}$ & $3867.31^{* *}$ & $0.83^{* *}$ & $53.20 \mathrm{~ns}$ & $1,811,853.7 \mathrm{~ns}$ \\
\hline $\mathrm{A} / \mathrm{F}$ & (27) & $89.35 * *$ & $12.60 * *$ & $52.71 * *$ & $2.79 * *$ & $1043.78^{* *}$ & $0.58^{* *}$ & $271,141.10^{* *}$ & $3,111,470.8 * *$ \\
\hline $\mathrm{C} \times \mathrm{A}$ & 28 & $5.27 \mathrm{~ns}$ & $4.10 *$ & $23.68^{* *}$ & $1.92 * *$ & $954.65^{* *}$ & $0.02 \mathrm{~ns}$ & $157,755.84^{* *}$ & $2,816,928.8$ ** \\
\hline Error & 113 & 4.18 & 2.39 & 5.19 & 0.71 & 26.45 & 0.03 & $37,725.85$ & $937,710.7$ \\
\hline CV (\%) & & 15.9 & 34.95 & 22.22 & 31.44 & 22.52 & 17.42 & 47.28 & 62.64 \\
\hline Mean & & 12.87 & 4.38 & 10.36 & 2.65 & 23.68 & 1.07 & 433.92 & 1603.36 \\
\hline$\sigma_{g}^{2}$ & & 15.85 & 1.52 & 20.65 & 1.57 & 12.57 & 0.09 & $17,283.90$ & $41,354.50$ \\
\hline$\sigma_{g c}^{2}$ & & 0.46 & 0.69 & 6.53 & 0.44 & 328.04 & 0.00 & 40,010 & 626,406 \\
\hline$\sigma^{2}{ }_{e}$ & & 4.14 & 2.31 & 5.17 & 0.68 & 26.42 & 0.03 & $37,725.80$ & $937,710.70$ \\
\hline$\sigma_{p}^{2}$ & & 20.45 & 4.52 & 32.35 & 2.69 & 367.03 & 0.12 & $95,019.70$ & $1,605,471.20$ \\
\hline
\end{tabular}

Note ${ }^{*}, * *$, ns: significant at $p \leq 0.05, p \leq 0.01$ and non-significant, respectively, $\mathrm{DF}=$ degree of freedom at $0.05, \mathrm{CV}(\%)=$ coefficient of variation $(\%), \sigma^{2} \mathrm{~g}=$ genotypic variance, $\sigma^{2}$ gc $=$ genotype $\mathrm{x}$ cropping condition variance, $\sigma^{2}=$ error variance, $\sigma^{2} p=$ phenotypic variance, FGI $=$ fruit girth $(\mathrm{cm})$, DFR = diameter of fruit $(\mathrm{cm})$, FLE $=$ fruits length $(\mathrm{cm})$, FLW $=$ fruits length to width ratio (no.), $\mathrm{TNF}=$ total number of fruits (no.), NFB = number of fruits per bunch (no.), AFW = average fruits weight $(\mathrm{g}), \mathrm{FYP}=$ fruit yield per plant $(\mathrm{g})$. 
The yield traits' least significant difference (LSD) mean performances of 29 accessions are presented in Table 3. The fruit girth (FGI) ranged from $22.37 \mathrm{~cm}$ to $5.71 \mathrm{~cm}$ with an average value of $12.84 \mathrm{~cm}$. Accession 13MR (Malaysian Round) had the longest fruit girth (FGI), while accession 6TL (Thailand Long) showed the smallest fruit girth (FGI). Concurrently, the same accession pattern was observed for the diameter of fruit (DFR) that showed an average diameter of $4.36 \mathrm{~cm}$. Accession 6TL recorded the smallest diameter of $1.99 \mathrm{~cm}$, and the widest diameter of fruit (DFR) was observed in 13MR at $7.31 \mathrm{~cm}$. The fruit length (FLE) varied from $2.10 \mathrm{~cm}$ to $17.70 \mathrm{~cm}$. The longest fruit length (FLE) was observed in 2TL, while accession 5TR (Thailand Round) had the shortest fruit length (FLE). The mean fruit length (FLE) among accessions was $10.31 \mathrm{~cm}$. For fruit length to width ratio (FLW), accession 1TR recorded the lowest with ratio (0.73), while a ratio of 5.15 was observed in 10ML (Malaysian Long) with an average mean of 2.65. The highest total number of fruits (TNF) was produced by 15ML (63.17), and accession 4TR produced the lowest number of fruits (TNF) at 4.50 fruits. The average total number of fruits (TNF) produced was 23.19 among the accessions. The average number of fruits per bunch (NFB) was 1.07 with 6TL producing the highest (2.67), and the remaining accession had the lowest number of fruits per bunch (NFB) (1.00) except for 26CL (China Long), which had an intermediate (1.40) total number of fruits per bunch (NFB). The average fruit weight (AFW) was $433.92 \mathrm{~g}$. The fruit weight ranged from $142.50 \mathrm{~g}$ (5TR) to $962.90 \mathrm{~g}$ (21ML). The overall yield per plant (FYP) means was 1603.36 g. The yield per plant (FYP) ranged from $323.90 \mathrm{~g}$ (4TR) to $2932.20 \mathrm{~g}$ (13MR). Generally, accession 13 MR had the best performance in fruit yield per plant (FYP) in both cropping conditions with slightly lower yield in glasshouse cropping conditions. Generally, mean comparisons of accession performances between two cropping condition portrayed a higher mean value (as indicated in bold) in the open field except for the number of fruits per bunch (NFB). Indeed, the yield is evergreen major parameters for evaluating cropping conditions. Pollination in the open field is more frequent, as it is aided with natural pollinators such as bees and wind flow to help dissemination and distribution of pollen. Meanwhile, in glasshouse conditions, limited aeration and higher temperature due to cladding materials in this microclimate [28] eventually can reduce the fruit set. Alternatively, hand pollination assistance through shaking flowers is seen as the savior for promoting the set of the first blossoms of the flowers, and this implicitly causes more labor work and requires higher costs. Other than that, eggplant is more susceptible to whiteflies family species (Aleyrodidae sp.) in a glasshouse compared to the open field. This might be due to higher temperatures causing an outbreak of whiteflies due to a thermal tolerance up to $40-45^{\circ} \mathrm{C}$ in the glasshouse [29]. 
Table 3. Means yield and yield traits studied in 29 eggplant accessions across two cropping conditions.

\begin{tabular}{|c|c|c|c|c|c|c|c|c|c|c|c|c|}
\hline \multirow[t]{2}{*}{ Accessions } & \multicolumn{3}{|c|}{ FGI $(\mathrm{cm})$} & \multicolumn{3}{|c|}{ DFR (cm) } & \multicolumn{3}{|c|}{ FLE (cm) } & \multicolumn{3}{|c|}{ FLW (ratio) } \\
\hline & OP & GH & Pooled & OP & GH & Pooled & OP & GH & Pooled & OP & GH & Pooled \\
\hline 1 TR & $13.41_{\mathrm{d}-\mathrm{g}}$ & $10.54_{c-e}$ & $11.97_{\mathrm{d}-\mathrm{h}}$ & $9.40_{\mathrm{ab}}$ & $3.38_{\mathrm{f}-\mathrm{i}}$ & $6.39_{\mathrm{abc}}$ & $4.09_{\mathrm{i}-\mathrm{k}}$ & $3.18_{\mathrm{kl}}$ & $3.63_{1}$ & $0.51_{\mathrm{k}}$ & $0.94_{\mathrm{kl}}$ & $0.73_{1}$ \\
\hline $2 \mathrm{TL}$ & $11.41_{f-h}$ & $10.67_{\mathrm{c}-\mathrm{e}}$ & $11.04_{\mathrm{e}-\mathrm{j}}$ & $6.89_{\mathrm{a}-\mathrm{g}}$ & $3.32_{\mathrm{f}-\mathrm{i}}$ & $5.11_{\mathrm{b}-\mathrm{e}}$ & $19.04_{\mathrm{ab}}$ & $16.37 \mathrm{a}$ & $17.70_{\mathrm{a}}$ & $3.66_{\mathrm{c}-\mathrm{g}}$ & $4.95_{\mathrm{ab}}$ & $4.31_{\mathrm{ab}}$ \\
\hline 3 TR & $16.09_{\mathrm{cd}}$ & $11.49_{\mathrm{cd}}$ & $13.79_{\mathrm{d}}$ & $5.02_{\mathrm{d}-\mathrm{i}}$ & $3.69_{\mathrm{f}-\mathrm{h}}$ & $4.36_{\mathrm{def}}$ & $5.23_{\mathrm{g}-\mathrm{k}}$ & $3.76_{j-1}$ & $4.49 \mathrm{kl}$ & $1.04_{\mathrm{i}-\mathrm{k}}$ & $1.02 \mathrm{kl}$ & $1.03_{\mathrm{kl}}$ \\
\hline 4 TR & $16.22_{\mathrm{cd}}$ & $16.44_{\mathrm{ab}}$ & $16.33_{\mathrm{c}}$ & $5.10_{\mathrm{d}-\mathrm{i}}$ & $5.09_{\mathrm{b}-\mathrm{e}}$ & $5.10_{\text {cde }}$ & $6.17_{\mathrm{f}-\mathrm{j}}$ & $7.40_{\mathrm{g}-j}$ & $6.79_{\mathrm{jk}}$ & $1.22_{\mathrm{i}-\mathrm{k}}$ & $1.47_{\mathrm{i}-1}$ & $1.34_{\mathrm{jkl}}$ \\
\hline $5 \mathrm{TR}$ & $6.87_{\mathrm{ij}}$ & $7.17_{\mathrm{ef}}$ & $7.02 \mathrm{kl}$ & $2.11_{\mathrm{i}}$ & $2.21_{i j}$ & $2.16_{\mathrm{gh}}$ & $2.06_{\mathrm{k}}$ & $2.13_{1}$ & $2.10_{1}^{n}$ & $0.98_{\mathrm{i}-\mathrm{k}}$ & $0.96_{\mathrm{kl}}$ & $0.97_{\mathrm{kl}}$ \\
\hline $6 \mathrm{TL}$ & $5.93_{j}$ & $5.49_{\mathrm{f}}$ & $5.71_{1}$ & $2.30_{i}$ & $1.67_{j}$ & $1.99_{\mathrm{h}}$ & $4.55_{\mathrm{h}-\mathrm{k}}$ & $4.63_{\mathrm{i}-1}$ & $4.59 \mathrm{kl}$ & $2.18_{\mathrm{g}-\mathrm{j}}$ & $2.79_{\mathrm{d}-\mathrm{h}}$ & $2.49_{\mathrm{f}-\mathrm{i}}$ \\
\hline $7 \mathrm{TR}$ & $14.56_{\mathrm{c}-\mathrm{e}}$ & $7.99_{\mathrm{d}-\mathrm{f}}$ & $11.28_{\mathrm{e}-\mathrm{i}}$ & $4.53_{\mathrm{e}-\mathrm{i}}$ & $3.50_{\mathrm{f}-\mathrm{h}}$ & $4.02_{\mathrm{def}}$ & $4.24_{\mathrm{h}-\mathrm{k}}$ & $3.21_{\mathrm{kl}}$ & $3.72_{1}$ & $0.94_{i-k}$ & $0.92_{\mathrm{kl}}$ & $0.93_{\mathrm{kl}}$ \\
\hline $8 \mathrm{ML}$ & $13.92_{c-f}$ & $11.60_{\mathrm{cd}}$ & $13.34_{\mathrm{de}}$ & $8.52_{a-c}$ & $3.55_{\mathrm{f}-\mathrm{h}}$ & $7.28_{a}$ & $18.43_{\mathrm{ab}}$ & $13.20_{\mathrm{a}-\mathrm{d}}$ & $17.12 \mathrm{ab}$ & $3.21_{\mathrm{d}-\mathrm{h}}$ & $3.72_{\mathrm{b}-\mathrm{e}}$ & $3.34_{b-f}$ \\
\hline $9 \mathrm{ML}$ & $13.25_{\mathrm{d}-\mathrm{g}}$ & $9.65_{c-e}$ & $12.05_{\mathrm{d}-\mathrm{g}}$ & $4.41_{\mathrm{e}-\mathrm{i}}$ & $2.99_{\mathrm{f}-\mathrm{h}}$ & $3.94_{\mathrm{d}-\mathrm{g}}$ & $20.11_{a}$ & $11.24_{\mathrm{b}-\mathrm{g}}$ & $17.15 \mathrm{ab}$ & $4.58 \mathrm{a}-\mathrm{e}$ & $3.76_{a-d}$ & $4.30_{\mathrm{ab}}$ \\
\hline $10 \mathrm{ML}$ & $12.52 \mathrm{e}-\mathrm{h}$ & $9.02_{\mathrm{d}-\mathrm{f}}$ & $10.77_{f-j}$ & $3.82_{\mathrm{g}-\mathrm{i}}$ & $2.83_{g-j}$ & $3.32_{\mathrm{e}-\mathrm{h}}$ & $20.25 a$ & $14.14_{\mathrm{ab}}$ & $17.19 \mathrm{ab}$ & $5.32 \mathrm{ab}$ & $4.98_{\mathrm{a}}$ & $5.15_{a}$ \\
\hline $13 \mathrm{MR}$ & $24.39 \mathrm{a}$ & $20.36_{a}$ & $22.37 a$ & $8.12_{\mathrm{a}-\mathrm{d}}$ & $6.50_{a}$ & $7.31_{a}$ & $7.94_{f-h}$ & $6.69_{\mathrm{h}-\mathrm{k}}$ & $7.31_{\mathrm{j}}$ & $0.98_{\mathrm{i}-\mathrm{k}}$ & $1.03_{\mathrm{k} l}$ & $1.00_{\mathrm{kl}}$ \\
\hline $14 \mathrm{ML}$ & $10.39_{\mathrm{gh}}$ & $7.91_{\mathrm{d}-\mathrm{f}}$ & $9.15_{\mathrm{ijk}}$ & $3.47_{\mathrm{hi}}$ & $2.48_{\mathrm{h}-\mathrm{j}}$ & $2.98_{\mathrm{fgh}}$ & $16.03_{\mathrm{bc}}$ & $8.03_{\mathrm{f}-\mathrm{i}}$ & $12.03 \mathrm{fgh}$ & $4.65_{a-e}$ & $3.28_{\mathrm{c}-\mathrm{g}}$ & $3.96_{\mathrm{bcd}}$ \\
\hline $15 \mathrm{ML}$ & $11.82_{\mathrm{e}-\mathrm{h}}$ & $10.78_{\mathrm{c}-\mathrm{e}}$ & $11.30_{\mathrm{e}-\mathrm{i}}$ & $3.77_{\mathrm{g}-\mathrm{i}}$ & $3.45_{\mathrm{f}-\mathrm{h}}$ & $3.61_{\mathrm{d}-\mathrm{h}}$ & $3.94_{j k}$ & $2.62_{\mathrm{kl}}$ & 3.28 & $\begin{array}{l}1.07 \mathrm{i}-\mathrm{k} \\
\text { in }\end{array}$ & $0.76_{1}$ & $0.91_{\mathrm{kl}}$ \\
\hline $16 \mathrm{ML}$ & $10.38_{\mathrm{gh}}$ & $8.73_{\mathrm{d}-\mathrm{f}}$ & $9.56_{\mathrm{h}-\mathrm{i}}$ & $3.60_{\mathrm{g}-\mathrm{i}}$ & $2.73_{\mathrm{h}-\mathrm{j}}$ & $3.17_{\text {fgh }}$ & $17.09_{\mathrm{ab}}^{\mathrm{K}}$ & $6.66_{\mathrm{h}-\mathrm{k}}$ & $11.88_{\mathrm{fgh}}$ & $4.799_{a-d}$ & $2.51_{\mathrm{e}-\mathrm{i}}$ & $3.65_{\mathrm{b}-\mathrm{e}}$ \\
\hline $17 \mathrm{ML}$ & $12.87_{\mathrm{e}-\mathrm{g}}$ & $13.15_{b c}$ & $12.98_{\mathrm{def}}$ & $3.89_{\mathrm{g}-\mathrm{i}}$ & $4.08_{\mathrm{d}-\mathrm{f}}$ & $3.97_{\mathrm{d}-\mathrm{g}}$ & $17.27_{\mathrm{ab}}$ & $11.00_{\mathrm{b}-\mathrm{g}}$ & $14.76_{\mathrm{b}-\mathrm{e}}$ & $4.45_{a-e}$ & $2.53_{d-i}$ & $3.68_{\mathrm{b}-\mathrm{e}}$ \\
\hline $18 \mathrm{ML}$ & $13.29_{\mathrm{d}-\mathrm{g}}$ & $13.25_{\mathrm{bc}}$ & $13.27_{\mathrm{de}}$ & $3.94_{\mathrm{g}-\mathrm{i}}^{\mathrm{g}-1}$ & $4.13_{\mathrm{c}-\mathrm{f}}$ & $4.03_{\text {def }}$ & $18.09_{\mathrm{ab}}$ & $9.53_{c-h}$ & $13.81_{c-f}$ & $4.53_{\mathrm{a}-\mathrm{e}}$ & $2.29_{\mathrm{f}-\mathrm{j}}$ & $3.41_{b-f}$ \\
\hline $19 \mathrm{ML}$ & $13.30_{\mathrm{d}-\mathrm{g}}$ & $11.75_{\mathrm{cd}}$ & $12.53_{\mathrm{d}-\mathrm{g}}$ & $4.18_{\mathrm{e}-\mathrm{i}}$ & $3.65_{\mathrm{f}-\mathrm{h}}$ & $3.92_{\mathrm{d}-\mathrm{g}}$ & $12.72_{c-\mathrm{e}}$ & $17.07 \mathrm{a}$ & $14.89 \mathrm{~b}-\mathrm{e}$ & $3.37_{\mathrm{d}-\mathrm{g}}$ & $4.74_{\mathrm{ab}}$ & $4.05_{\mathrm{bc}}$ \\
\hline $20 \mathrm{ML}$ & $11.48_{\mathrm{f}-\mathrm{h}}$ & $11.33_{\mathrm{cd}}$ & $11.40_{\mathrm{d}-\mathrm{i}}$ & $3.54_{\mathrm{g}-\mathrm{i}}$ & $3.47_{\mathrm{f}-\mathrm{h}}$ & $3.50_{d-h}$ & $16.08_{b c}$ & $10.86_{b-g}$ & $13.47_{\mathrm{d}-\mathrm{g}}$ & $4.60-\mathrm{a}-\mathrm{e}$ & $3.16_{c-g}$ & $3.88 \mathrm{bcd}$ \\
\hline $21 \mathrm{ML}$ & $24.0 a_{a}$ & $17.71_{\mathrm{a}}$ & $20.86_{a b}$ & $7.35_{\mathrm{a}-\mathrm{f}}$ & $5.32-a-c$ & $6.34_{a b c}$ & $8.49_{\mathrm{f}-\mathrm{g}}$ & $9.25_{d-h}$ & $8.87_{\mathrm{ij}}$ & $1.16_{i-k}$ & $2.12_{\mathrm{g}-\mathrm{k}}$ & $1.64_{i-1}$ \\
\hline $22 \mathrm{ML}$ & $23.69 \mathrm{a}$ & $20.20 \mathrm{a}$ & $22.29 a$ & $7.40 \mathrm{a}-\mathrm{e}$ & $6.08_{\mathrm{ab}}$ & $6.87_{\mathrm{abc}}$ & $13.09_{\mathrm{cd}}$ & $11.33_{\mathrm{b}-\mathrm{g}}$ & $12.38 \mathrm{efg}$ & $1.78_{\mathrm{h}-\mathrm{k}}$ & $1.86_{\mathrm{h}-1}$ & $1.81_{\mathrm{h}-\mathrm{k}}$ \\
\hline $23 \mathrm{ML}$ & $19.85_{b}$ & $19.43_{a}$ & $19.64_{b}$ & $6.27_{b-h}$ & $6.29_{\mathrm{ab}}$ & $6.28_{\mathrm{abc}}$ & $7.68_{\mathrm{f}-\mathrm{i}}$ & $6.60_{\mathrm{h}-\mathrm{k}}$ & $7.14 \mathrm{jk}$ & $1.22_{\mathrm{i}-\mathrm{k}}$ & $1.05_{j-1}$ & $1.14_{\mathrm{jkl}}$ \\
\hline $25 \mathrm{ML}$ & $16.59_{\mathrm{c}}$ & $16.72_{\mathrm{ab}}$ & $16.66_{\mathrm{c}}$ & $5.18_{\mathrm{c}-\mathrm{i}}$ & $5.18_{\mathrm{b}-\mathrm{d}}$ & $5.18_{\mathrm{bcd}}$ & $12.58_{c-e}$ & $9.42_{\mathrm{d}-\mathrm{h}}$ & $11.00 \mathrm{ghi}$ & $2.42_{\mathrm{f}-\mathrm{i}}$ & $1.83_{\mathrm{h}-1}$ & $2.12 \mathrm{~g}-\mathrm{j}$ \\
\hline $26 \mathrm{CL}$ & $9.52_{\mathrm{hi}}$ & $8.06_{\mathrm{d}-\mathrm{f}}$ & $8.64_{j k}$ & $3.00_{\mathrm{hi}}$ & $2.56_{\mathrm{h}-\mathrm{j}}$ & $2.73_{\mathrm{fgh}}$ & $9.32_{\text {ef }}$ & $7.50_{\mathrm{f}-\mathrm{j}}$ & $8.23_{j}$ & $3.11_{\mathrm{e}-\mathrm{h}}$ & $2.89_{\mathrm{c}-\mathrm{h}}$ & $2.97_{\mathrm{d}-\mathrm{g}}^{\mathrm{J}}$ \\
\hline $27 \mathrm{CL}$ & $13.02_{\mathrm{e}-\mathrm{g}}$ & $11.52_{\mathrm{cd}}$ & $12.27_{\mathrm{d}-\mathrm{g}}$ & $4.01_{\mathrm{f}-\mathrm{i}}$ & $3.50_{\mathrm{f}-\mathrm{h}}$ & $3.75_{\mathrm{d}-\mathrm{h}}$ & $20.61_{a}$ & $11.64_{\mathrm{b}-\mathrm{f}}$ & $16.13 \mathrm{a}-\mathrm{d}$ & $5.14_{\mathrm{a}-\mathrm{c}}$ & $3.49_{\mathrm{c}-\mathrm{f}}$ & $4.32_{\mathrm{ab}}$ \\
\hline $29 \mathrm{MN}$ & $11.57_{\text {e-h }}$ & $10.69_{\mathrm{c}-\mathrm{e}}$ & $11.13_{\mathrm{e}-\mathrm{i}}$ & $3.61_{\mathrm{g}-\mathrm{i}}$ & $3.35_{\mathrm{f}-\mathrm{i}}$ & $3.48_{\mathrm{d}-\mathrm{h}}$ & $12.93_{\mathrm{c}-\mathrm{e}}$ & $13.65_{a-c}$ & $13.29 \mathrm{efg}$ & $3.56_{\mathrm{c}-\mathrm{g}}$ & $4.09_{\mathrm{a}-\mathrm{c}}$ & $3.82_{\mathrm{bcd}}$ \\
\hline $30 \mathrm{MN}$ & $11.52_{\mathrm{e}-\mathrm{h}}$ & $10.91_{\mathrm{c}-\mathrm{e}}$ & $11.27_{\mathrm{e}-\mathrm{i}}$ & $3.59_{\mathrm{g}-\mathrm{i}}$ & $3.44_{f-h}$ & $3.53_{\mathrm{d}-\mathrm{h}}$ & $2.82_{\mathrm{jk}}$ & $2.60_{\mathrm{kl}}$ & $2.73_{1}$ & $0.79_{j k}$ & $0.76_{1}$ & $0.77_{1}$ \\
\hline $32 \mathrm{MN}$ & $10.46_{\mathrm{gh}}$ & $10.62_{c-e}$ & $10.54_{\mathrm{g}-\mathrm{j}}$ & $3.09_{\mathrm{hi}}$ & $3.04_{\mathrm{f}-\mathrm{i}}$ & $3.07_{\mathrm{fgh}}$ & $12.23_{\mathrm{de}}$ & $6.41_{\mathrm{h}-\mathrm{k}}$ & $9.32 \mathrm{hij}$ & $3.99_{b-f}$ & $2.13_{g-k}$ & $3.06_{c-g}$ \\
\hline $34 \mathrm{CL}$ & $11.79_{\mathrm{e}-\mathrm{h}}$ & $11.10_{\mathrm{c}-\mathrm{e}}$ & $11.45_{\mathrm{d}-\mathrm{i}}$ & $3.58_{\mathrm{g}-\mathrm{i}}$ & $3.33_{\mathrm{f}-\mathrm{i}}$ & $3.45_{\mathrm{d}-\mathrm{h}}$ & $20.18 a$ & $8.50_{\mathrm{e}-\mathrm{i}}$ & $14.34_{\mathrm{c}-\mathrm{f}}$ & $5.70_{a}$ & $2.56_{\mathrm{d}-\mathrm{i}}$ & $4.13_{b}$ \\
\hline $35 \mathrm{CL}$ & $14.33_{\mathrm{c}-\mathrm{f}}$ & $11.83_{\mathrm{cd}}$ & $13.08_{\mathrm{def}}$ & $9.93_{a}$ & $3.95 \mathrm{e}-\mathrm{g}$ & $6.94_{\mathrm{ab}}$ & $20.40_{a}$ & $12.19_{\mathrm{b}-\mathrm{e}}$ & $16.29 \mathrm{abc}$ & $2.34_{\mathrm{g}-\mathrm{j}}$ & $3.20_{\mathrm{c}-\mathrm{g}}$ & $2.77_{\mathrm{e}-\mathrm{h}}$ \\
\hline Mean & 13.79 & 11.89 & 12.84 & 4.98 & 3.74 & 4.36 & 12.13 & 8.48 & 10.31 & 2.85 & 2.44 & 2.65 \\
\hline $\operatorname{LSD}(p=0.05)$ & 3.06 & 4.03 & 2.43 & 3.39 & 1.22 & 1.84 & 3.72 & 4.16 & 2.71 & 1.59 & 1.24 & 1.00 \\
\hline SEM & 0.06 & 0.05 & 0.06 & 0.03 & 0.02 & 0.03 & 0.08 & 0.06 & 0.07 & 0.02 & 0.02 & 0.02 \\
\hline
\end{tabular}


Table 3. Cont.

\begin{tabular}{|c|c|c|c|c|c|c|c|c|c|c|c|c|}
\hline \multirow[t]{2}{*}{ Accessions } & \multicolumn{3}{|c|}{ TNF (no.) } & \multicolumn{3}{|c|}{ NFB (no.) } & \multicolumn{3}{|c|}{ AFW (g) } & \multicolumn{3}{|c|}{ FYP (g) } \\
\hline & OP & GH & Pooled & OP & GH & Pooled & OP & GH & Pooled & OP & GH & Pooled \\
\hline $1 \mathrm{TR}$ & $19.00_{m-p}$ & $10.67_{\mathrm{ef}}$ & $14.83_{\mathrm{klm}}$ & $1.00_{\mathrm{b}}$ & $1.00_{\mathrm{c}}$ & $1.00_{c}$ & $315.90_{j-m}$ & $87.76_{b c}$ & $201.80_{\mathrm{i}}$ & $974.13_{\mathrm{i}-\mathrm{k}}$ & $190.57_{c-h}$ & $582.30_{\text {hij }}$ \\
\hline $2 \mathrm{TL}$ & $37.33_{\mathrm{g}-\mathrm{i}}$ & $4.00_{\mathrm{i}-\mathrm{m}}$ & $20.67_{\mathrm{h}-\mathrm{k}}$ & $1.00_{\mathrm{b}}$ & $1.00_{\mathrm{c}}$ & $1.00_{\mathrm{c}}$ & $767.70_{d-h}$ & $181.90_{\mathrm{bc}}$ & $474.80_{\mathrm{d}-\mathrm{g}}$ & $3317.28 \mathrm{a}-\mathrm{g}$ & $207.23_{\mathrm{c}-\mathrm{h}}$ & $1762.30_{b-f}$ \\
\hline $3 \mathrm{TR}$ & $71.00_{\mathrm{c}}$ & $20.33_{c}$ & $45.67_{\mathrm{b}}$ & $1.00_{\mathrm{b}}$ & $1.00_{\mathrm{c}}$ & $1.00_{\mathrm{c}}$ & $944.58_{\mathrm{c}-\mathrm{e}}$ & $249.08_{b c}$ & $596.80_{\mathrm{b}-\mathrm{e}}$ & $4556.22 \mathrm{a}-\mathrm{d}$ & $498.15_{b-d}$ & $2527.20_{\mathrm{abc}}$ \\
\hline $4 \mathrm{TR}$ & $3.33_{\mathrm{q}}$ & $5.67_{\mathrm{g}-1}$ & $4.50_{\mathrm{o}}$ & $1.00_{\mathrm{b}}$ & $1.00_{\mathrm{c}}$ & $1.00 \mathrm{c}$ & $116.89 \mathrm{~m}$ & $214.50_{\mathrm{bc}}$ & $165.70_{\mathrm{i}}$ & $218.70_{\mathrm{k}}$ & $429.10_{b-f}$ & $323.90_{j}$ \\
\hline $5 \mathrm{TR}$ & $27.00_{\mathrm{i}-\mathrm{n}}$ & $45.67 a$ & $36.33_{\mathrm{cd}}$ & $2.67 \mathrm{a}$ & $2.67 \mathrm{a}$ & $2.67 \mathrm{a}$ & $185.88_{\mathrm{lm}}$ & $99.21_{b c}$ & $142.50_{\mathrm{i}}$ & $811.47_{j k}$ & $198.40_{\mathrm{c}-\mathrm{h}}$ & $504.90_{\mathrm{ij}}$ \\
\hline $6 \mathrm{TL}$ & $29.33_{h-m}$ & $26.33_{b}$ & $27.83_{\mathrm{ef}}$ & $1.00_{\mathrm{b}}$ & $1.00_{\mathrm{c}}$ & $1.00_{c}$ & $212.78_{1 \mathrm{~m}}$ & $101.83_{\mathrm{bc}}$ & $157.30_{\mathrm{i}}$ & $937.06_{i-k}$ & $136.35 \mathrm{~d}-\mathrm{h}$ & $536.70 \mathrm{ij}$ \\
\hline $7 \mathrm{TR}$ & $35.33_{\mathrm{g}-\mathrm{k}}$ & $20.67_{c}$ & $28.00_{\mathrm{e}}$ & $1.00_{\mathrm{b}}$ & $1.00_{\mathrm{c}}$ & $1.00 \mathrm{c}$ & $370.56_{i-m}$ & $201.57_{b c}$ & $286.10 \mathrm{ghi}$ & $1812.47_{\mathrm{g}-\mathrm{k}}$ & $387.6_{\mathrm{b}-\mathrm{g}}$ & $1100.00_{f-j}$ \\
\hline $8 \mathrm{ML}$ & $17.67_{n-p}$ & $1.00_{\mathrm{m}}$ & $13.50_{\mathrm{lmn}}$ & $1.00_{\mathrm{b}}$ & $1.00_{\mathrm{c}}$ & $1.00_{\mathrm{c}}$ & $379.53_{\mathrm{i}-\mathrm{m}}$ & $26.00_{c}$ & $202.80_{\mathrm{i}}$ & $2996.47_{b-j}$ & $26.00_{\mathrm{gh}}^{\circ}$ & $1511.20_{\mathrm{c}-\mathrm{i}}$ \\
\hline $9 \mathrm{ML}$ & $21.00_{m-p}$ & $5.00_{\mathrm{h}-\mathrm{m}}$ & $15.67_{j-m}$ & $1.00_{\mathrm{b}}$ & $1.00_{\mathrm{c}}$ & $1.00 \mathrm{c}$ & $862.45_{d-f}$ & $108.25_{b c}$ & $485.40_{\mathrm{d}-\mathrm{g}}$ & $2300.37_{\mathrm{e}-\mathrm{k}}$ & $108.20_{\mathrm{e}-\mathrm{h}}^{\circ}$ & $1204.30_{\mathrm{g}-\mathrm{j}}$ \\
\hline $10 \mathrm{ML}$ & $40.00_{f-h}$ & $16.67_{\mathrm{cd}}$ & $28.33_{\mathrm{e}}$ & $1.00_{\mathrm{b}}$ & $1.00_{\mathrm{c}}$ & $1.00_{\mathrm{c}}$ & $1248.01_{\mathrm{ab}}$ & $338.89_{b c}$ & $793.40 \mathrm{ab}$ & $5049.57_{\mathrm{ab}}$ & $677.77_{\mathrm{b}}$ & $2863.70_{\mathrm{ab}}$ \\
\hline $13 \mathrm{MR}$ & $32.00_{\mathrm{g}-1}$ & $5.00_{\mathrm{h}-\mathrm{m}}$ & $18.50_{\mathrm{i}-1}$ & $1.00_{\mathrm{b}}$ & $1.00_{\mathrm{c}}$ & $1.00 \mathrm{c}$ & $1027.82_{b-d}$ & $295.43_{b c}$ & $661.60_{\mathrm{bcd}}$ & $5342.90_{a}$ & $521.59_{\mathrm{bc}}$ & $2932.20 \mathrm{a}$ \\
\hline $14 \mathrm{ML}$ & $64.00_{\mathrm{cd}}$ & $10.67_{\text {ef }}$ & $37.33_{c}$ & $1.00_{\mathrm{b}}$ & $1.00_{\mathrm{c}}$ & $1.00 \mathrm{c}$ & $926.75_{c-e}$ & $127.43_{\mathrm{bc}}$ & $527.10_{\text {def }}$ & $4467.10_{\mathrm{a}-\mathrm{e}}$ & $254.86_{c-h}$ & $2361.00_{a-d}$ \\
\hline $15 \mathrm{ML}$ & $116.33_{a}$ & $10.00_{\mathrm{e}-\mathrm{g}}$ & $63.17 \mathrm{a}$ & $1.00_{\mathrm{b}}$ & $1.00_{\mathrm{c}}$ & $1.00 \mathrm{c}$ & $483.21_{h-1}$ & $101.36_{\mathrm{bc}}$ & $292.30 \mathrm{ghi}$ & $2462.54_{\mathrm{d}-\mathrm{j}}$ & $180.66_{\mathrm{c}-\mathrm{h}}$ & $1321.60_{\mathrm{d}-\mathrm{j}}$ \\
\hline $16 \mathrm{ML}$ & $60.00_{\mathrm{de}}$ & $2.00_{\mathrm{k}-\mathrm{m}}$ & $31.00_{\mathrm{de}}$ & $1.00_{\mathrm{b}}$ & $1.00_{\mathrm{c}}$ & $1.00_{c}$ & $933.33_{c-e}$ & $61.35_{\mathrm{bc}}$ & $497.30_{\mathrm{d}-\mathrm{g}}^{\circ}$ & $4666.66_{a-d}$ & $61.35_{\mathrm{f}-\mathrm{h}}$ & $2364.00_{a-d}$ \\
\hline $17 \mathrm{ML}$ & $25.00_{\mathrm{k}-\mathrm{o}}$ & $3.00_{j-m}$ & $16.20_{\mathrm{i}-1}$ & $1.00_{\mathrm{b}}$ & $1.00_{\mathrm{c}}$ & $1.00 \mathrm{c}$ & $821.03_{\mathrm{d}-\mathrm{g}}$ & $111.47_{\mathrm{bc}}$ & $466.30_{\mathrm{d}-\mathrm{g}}$ & $3750.81_{\mathrm{a}-\mathrm{g}}$ & $111.47_{\mathrm{e}-\mathrm{h}}$ & $1931.10 \mathrm{a}-\mathrm{f}$ \\
\hline $18 \mathrm{ML}$ & $27.67_{\mathrm{i}-\mathrm{n}}$ & $8.33_{\mathrm{f}-\mathrm{i}}$ & $18.00_{\mathrm{i}-1}$ & $1.00_{\mathrm{b}}$ & $1.00_{\mathrm{c}}$ & $1.00_{\mathrm{c}}$ & $762.35_{\mathrm{d}-\mathrm{h}}$ & $206.19 \mathrm{bc}$ & $484.30_{\mathrm{d}-\mathrm{g}}$ & $3742.40_{a-g}$ & $348.32_{b-h}$ & $2045.40_{a-f}$ \\
\hline $19 \mathrm{ML}$ & $12.67 \mathrm{pq}$ & $2.33_{\mathrm{k}-\mathrm{m}}$ & $7.50 \mathrm{mno}$ & $1.00_{\mathrm{b}}$ & $1.00_{\mathrm{c}}$ & $1.00_{c}$ & $476.10_{\mathrm{h}-1}$ & $111.29 \mathrm{bc}$ & $293.70_{\mathrm{ghi}}$ & $1785.35_{\mathrm{g}-\mathrm{k}}$ & $222.58_{\mathrm{c}-\mathrm{h}}$ & $1004.00_{\mathrm{f}-\mathrm{j}}$ \\
\hline $20 \mathrm{ML}$ & $40.67_{f g}$ & $13.00_{\mathrm{de}}$ & $26.83_{\mathrm{efg}}$ & $1.00_{\mathrm{b}}$ & $1.00_{\mathrm{c}}$ & $1.00 \mathrm{c}$ & $891.35_{\mathrm{de}}$ & $242.33_{\mathrm{bc}}$ & $566.80_{c-f}$ & $3779.90 \mathrm{a}-\mathrm{g}$ & $484.66_{\mathrm{b}-\mathrm{e}}$ & $2132.30 \mathrm{a}-\mathrm{e}$ \\
\hline $21 \mathrm{ML}$ & $25.67_{j-n}$ & $6.00_{\mathrm{g}-1}$ & $15.83_{\mathrm{i}-\mathrm{m}}$ & $1.00_{\mathrm{b}}$ & $1.00_{\mathrm{c}}$ & $1.00 \mathrm{c}$ & $981.66_{b-d}$ & $944.21_{\mathrm{a}}$ & $962.90 \mathrm{ab}$ & $2225.11_{\mathrm{f}-\mathrm{k}}$ & $1208.66_{a}$ & $1716.90_{\mathrm{c}-\mathrm{g}}$ \\
\hline $22 \mathrm{ML}$ & $22.67_{1-p}$ & $4.33_{\mathrm{h}-\mathrm{m}}$ & $13.50_{\mathrm{lmn}}$ & $1.00_{\mathrm{b}}$ & $1.00_{\mathrm{c}}$ & $1.00 \mathrm{c}$ & $1194.09_{\mathrm{a}-\mathrm{c}}$ & $99.27_{b c}$ & $646.70_{\mathrm{b}-\mathrm{e}}$ & $4205.55_{a-f}$ & $198.54_{\mathrm{c}-\mathrm{h}}$ & $2202.00_{a}-\mathrm{e}$ \\
\hline $23 \mathrm{ML}$ & $24.00_{1-\mathrm{o}}$ & $1.67_{\mathrm{lm}}$ & $12.83 \mathrm{lmn}$ & $1.00_{\mathrm{b}}$ & $1.00_{\mathrm{c}}$ & $1.00 \mathrm{c}$ & $1397.98_{a}$ & $163.49_{\mathrm{bc}}$ & $780.70_{\mathrm{abc}}$ & $4674.73_{\mathrm{a}-\mathrm{c}}$ & $209.87_{c-h}$ & $2442.30_{\mathrm{abc}}$ \\
\hline $25 \mathrm{ML}$ & $14.67_{\text {op }}$ & $8.67_{\mathrm{e}-\mathrm{h}}$ & $11.67 \mathrm{mn}$ & $1.00_{\mathrm{b}}$ & $1.00_{\mathrm{c}}$ & $1.00 \mathrm{c}$ & $587.80_{f-j}$ & $3.72_{c}$ & 295.80 ghi & $2284.97_{\mathrm{e}-\mathrm{k}}$ & $5.84_{\mathrm{h}}$ & $1145.40_{\mathrm{f}-\mathrm{j}}$ \\
\hline $26 \mathrm{CL}$ & $49.33_{\text {ef }}$ & $3.67_{j-m}$ & $26.50_{\mathrm{e}-\mathrm{h}}$ & $1.00_{\mathrm{b}}$ & $1.67_{\mathrm{b}}$ & $1.40_{\mathrm{b}}$ & $270.86_{k-m}$ & $165.8_{\mathrm{bc}}$ & $218.30 \mathrm{hi}$ & $992.46_{h-k}$ & $283.74_{\mathrm{c}-\mathrm{h}}$ & $638.10_{g-j}$ \\
\hline $27 \mathrm{CL}$ & $18.67_{m-p}$ & $7.00_{\mathrm{f}-\mathrm{j}}$ & $12.83_{\mathrm{lmn}}$ & $1.00_{\mathrm{b}}$ & $1.00_{\mathrm{c}}$ & $1.00_{\mathrm{c}}$ & $572.50_{f-j}$ & $377.64_{\mathrm{b}}$ & $475.10_{\mathrm{d}-\mathrm{g}}$ & $3199.05_{a-h}$ & $377.64_{\mathrm{b}-\mathrm{h}}$ & $1788.30_{\mathrm{b}-\mathrm{f}}$ \\
\hline $29 \mathrm{MN}$ & $36.00_{g-j}$ & $6.33_{\mathrm{f}-\mathrm{k}}$ & $21.17_{g-j}$ & $1.00_{\mathrm{b}}$ & $1.00_{\mathrm{c}}$ & $1.00_{c}$ & $546.08_{\mathrm{g}-\mathrm{k}}$ & $167.38_{\mathrm{bc}}$ & $356.70_{f-i}$ & $2736.63_{c-j}$ & $290.92_{\mathrm{c}-\mathrm{h}}$ & $1513.80_{c-i}$ \\
\hline $30 \mathrm{MN}$ & $96.00_{\mathrm{b}}$ & $4.00_{\mathrm{i}-\mathrm{k}}$ & $59.20_{a}$ & $1.00_{\mathrm{b}}$ & $1.00_{\mathrm{c}}$ & $1.00_{\mathrm{c}}$ & $462.399_{\mathrm{i}-1}$ & $19.66_{c}$ & $241.00_{\mathrm{h}-\mathrm{i}}$ & $2311.94_{\mathrm{e}-\mathrm{k}}$ & $39.32_{\mathrm{gh}}$ & $1175.60_{f-j}$ \\
\hline $32 \mathrm{MN}$ & $37.33_{g-i}$ & $6.33_{\mathrm{f}-\mathrm{k}}$ & $21.83_{\mathrm{f}-\mathrm{i}}$ & $1.00_{\mathrm{b}}$ & $1.00_{\mathrm{c}}$ & $1.00 \mathrm{c}$ & $582.65_{f-j}$ & $121.85_{\mathrm{bc}}$ & $352.30_{\mathrm{f}-\mathrm{i}}$ & $3098.90_{b-i}$ & $201.09_{\mathrm{c}-\mathrm{h}}$ & $1650.00_{\mathrm{c}-\mathrm{h}}$ \\
\hline $34 \mathrm{CL}$ & $33.33_{g-1}$ & $9.67 \mathrm{e}-\mathrm{g}$ & $21.50_{g-j}$ & $1.00_{\mathrm{b}}$ & $1.00_{\mathrm{c}}$ & $1.00_{\mathrm{c}}$ & $656.63_{e-i}$ & $197.53_{\mathrm{bc}}$ & $427.10 \mathrm{e}-\mathrm{h}$ & $2959.85_{b-j}$ & $382.49_{b-h}$ & 1671.20 \\
\hline $35 \mathrm{CL}$ & $19.67_{m-p}$ & $7.00_{\mathrm{f}-\mathrm{j}}$ & $13.33_{\mathrm{Imn}}$ & $1.00_{\mathrm{b}}$ & $1.00_{c}$ & $1.00 \mathrm{c}$ & $841.95_{\mathrm{d}-\mathrm{g}}$ & $220.15_{b c}$ & $531.10_{\mathrm{def}}$ & $2651.15_{c-j}$ & $440.30_{\mathrm{b}-\mathrm{e}}$ & $\begin{array}{c}\mathrm{c}-\mathrm{h} \\
1545.70_{\mathrm{c}-\mathrm{h}}\end{array}$ \\
\hline Mean & 36.44 & 9.95 & 23.19 & 1.06 & 1.08 & 1.07 & 683.48 & 184.36 & 433.92 & 2907.30 & 299.42 & 1603.36 \\
\hline $\operatorname{LSD}(p=0.05)$ & 10.99 & 4.61 & 6.08 & 0.18 & 0.4 & 0.22 & 298.96 & 335.38 & 222.19 & 2207.90 & 377.7 & 1107.70 \\
\hline SEM & 0.29 & 0.12 & 0.21 & 0 & 0 & 0 & 4.19 & 2.81 & 3.50 & 20.62 & 3.57 & 12.10 \\
\hline
\end{tabular}

Note: $\overline{\mathrm{OP}}=$ open field, $\mathrm{GH}=$ glasshouse, $\mathrm{FGI}=$ fruit girth $(\mathrm{cm}), \mathrm{DFR}=$ diameter of fruit $(\mathrm{cm}), \mathrm{FLE}=$ fruits length $(\mathrm{cm})$, FLW = fruits length to width $(\mathrm{ratio}), \mathrm{TNF}=$ total no of fruits $(\mathrm{no}$ ), $\mathrm{NFB}=$ number of fruits per bunch (no.), AFW = average fruits weight $(\mathrm{g}), \mathrm{FYP}=$ fruit yield per plant $(\mathrm{g}), \mathrm{LSD}=$ least significant difference, $\mathrm{SEM}=$ standard error of mean, $\mathrm{n}$. $\mathrm{s}=$ not significant at $p>0.05$ and means with the same letter in each column also not significantly different at $5 \%$ probability level. 


\subsubsection{Vegetative Traits Across Two Cropping Conditions}

The combined analysis of variance for vegetative traits is presented in Table 4 . Highly significant differences $(p \leq 0.01)$ were observed among the accessions for number of primary branches (NPB), plant height (PHE), stem diameter (SDM), and days to first flowering (DFF), while non-significant differences were observed in plant spread (PSP). To emphasize, plant height is the most critical vegetative indicator of high yield, as postulated by [25]. Together with other vegetative parameters, these traits contributed by genetic makeup were implicitly influenced by the environment, especially cropping conditions. This was mainly due to limited sources of photosynthates partitioning to meet vigorous sink competition. Hence, the quota for yield was unfairly used by somatic cells growth, which resulted in luxurious vegetative development and obvious height. For fruit type, all vegetative parameters showed no significant differences except for the number of primary branches (NPB), where a highly significant difference was observed, and plant spread (PSP), which showed a significant difference at $p \leq 0.05$. The fruit type within accessions showed highly significant differences for all vegetative parameters except for plant spread (PSP), which illustrated no significant difference. While cropping conditions also indicated high significance $(p \leq 0.01)$ for number of primary branches (NPB), plant height (PHE) and plant spread (PSP) were vegetative components that had an impact on genetic variation; stem diameter (SDM) and days to first flowering (DFF) indicated no significant difference. On the other hand, the interaction between cropping conditions with the accession showed a highly significant difference $(p \leq 0.01)$ for the stem diameter (SDM) trait only, and the remaining number of primary branches (NPB), plant height (PHE), days to first flowering (DFF), and plant spread (PSP) eventually showed no significant difference. Moreover, the result implied that there was a highly significant difference $(p \leq 0.01)$ in replication effect within the cropping conditions, which were number of primary branches (NPB), plant height (PHE), and plant spread (PSP). However, days to first flowering (DFF) and stem diameter (SDM) showed no significant difference.

Table 4. Mean square of vegetative parameters of 29 eggplant accessions.

\begin{tabular}{|c|c|c|c|c|c|c|}
\hline Sources of Variation & DF & NPB & PHE & SDM & PSP & DFF \\
\hline Cropping condition (C) & 1 & $357.57 * *$ & $76049.97^{* *}$ & $0.00 \mathrm{~ns}$ & $36858.45^{* *}$ & $175.06 \mathrm{~ns}$ \\
\hline Replications within C (R/C) & 4 & $27.59 * *$ & $473.55^{* *}$ & $0.05 \mathrm{~ns}$ & $1490.84 * *$ & $294.64 \mathrm{~ns}$ \\
\hline Accessions (A) & 28 & $7.49^{* *}$ & $368.68^{* *}$ & $0.49^{* *}$ & $356.81 \mathrm{~ns}$ & $369.05^{* *}$ \\
\hline Fruit types (F) & (1) & $58.58 * *$ & $1.67 \mathrm{~ns}$ & $0.00 \mathrm{~ns}$ & $1349.57 *$ & $8.65 \mathrm{~ns}$ \\
\hline $\mathrm{A} / \mathrm{F}$ & $(27)$ & $5.46^{* *}$ & $399.06^{* *}$ & $0.50 * *$ & 307.33 ns & $381.71^{* *}$ \\
\hline $\mathrm{C} \times \mathrm{A}$ & 28 & $2.99 \mathrm{~ns}$ & $187.36 \mathrm{~ns}$ & $0.32 * *$ & $381.72 \mathrm{~ns}$ & $136.42 \mathrm{~ns}$ \\
\hline Error & 113 & 2.60 & 143.59 & 0.03 & 280.45 & 158.85 \\
\hline CV (\%) & & 24.59 & 13.52 & 10.13 & 18.52 & 14.25 \\
\hline Mean & & 7.58 & 92.09 & 1.68 & 96.75 & 88.81 \\
\hline$\sigma^{2}$ & & 0.79 & 32.06 & 0.03 & 0.00 & 37.81 \\
\hline$\sigma^{2}{ }_{g c}^{8}$ & & 0.09 & 17.83 & 0.09 & 23.13 & 0.00 \\
\hline$\sigma_{e}^{2}$ & & 2.62 & 143.04 & 0.03 & 285.71 & 153.80 \\
\hline$\sigma^{2} p$ & & 3.50 & 192.93 & 0.15 & 308.84 & 191.61 \\
\hline
\end{tabular}

Note $=*, * *$, ns: significant at $p \leq 0.05, p \leq 0.01$ and non-significant respectively, DF $=$ degree of freedom at 0.05 , $\mathrm{CV}(\%)=$ coefficient of variation $(\%), \sigma_{\mathrm{g}}^{2}=$ genotype variance, $\sigma_{\mathrm{gc}}^{2}=$ genotype $\times$ cropping condition variance, $\sigma_{\mathrm{e}}^{2}=$ error variance, $\sigma_{\mathrm{p}}^{2}=$ phenotypic variance, $\mathrm{NPB}=$ number of primary branches, $\mathrm{PHE}=$ plant height 90 days after tra nsplant $(\mathrm{cm})$, $\mathrm{SDM}=$ stem diameter 90 days after tra nsplant $(\mathrm{cm}), \mathrm{PSP}=$ plant spread 90 days after tra nsplant $(\mathrm{cm}), \mathrm{DFF}=$ days to first flowering (day). 
The mean comparison for vegetative traits is presented in Table 5 . The average number of primary branches (NPB) was 7.54 and ranged from 5.67 (19ML) to 10.50 (30MN) (Malaysian Native). Plant height (PHE) varied from $73.80 \mathrm{~cm}(26 \mathrm{CL})$ to $105.60 \mathrm{~cm}$ (4TR) with an average of $91.72 \mathrm{~cm}$. The average stem diameter (SDM) for all accessions was $1.68 \mathrm{~cm}$; the smallest stem diameter was observed in 14ML at $1.31 \mathrm{~cm}$, while the largest was recorded by $25 \mathrm{ML}$ at $2.83 \mathrm{~cm}$. For plant spread (PSP), 14ML had the smallest with $82.81 \mathrm{~cm}$, and 3TR recorded the largest plant spread at $116.14 \mathrm{~cm}$. The average plant spread (PSP) length was $96.50 \mathrm{~cm}$. For days to flowering (DFF), 3TR recorded the longest days to flowering with 100.67 days, while 35CL recorded the earliest at 71.17 days. The average mean of days to flowering (DFF) was 88.81 days. Given the resulting comparison of accession mean performance for two cropping conditions, as shown in Table 5 , the means for all vegetative traits were comparatively higher in the greenhouse compared to the open field cropping conditions except for day for first flowering (DFF). This showed robust growth of vegetative yield in the greenhouse cropping condition that may be affected by a significant and continuous supply of fertilizer using irrigation. The variation of vegetative growth among eggplant accessions was wide enough to indicate the perspective glass view for improving accessions studied for all characteristics that eventually support and prepare the reproductive phase of eggplant. It was evidenced that this might be due to the association of genetic composition together with the environment factor applied. 
Table 5. Mea ns for vegetative characteristics studied in 29 accessio ns of eggplant across two cropping conditio ns.

\begin{tabular}{|c|c|c|c|c|c|c|c|c|c|c|c|c|c|c|c|}
\hline \multirow[t]{2}{*}{ Accession } & \multicolumn{3}{|c|}{ NPB (no.) } & \multicolumn{3}{|c|}{ PHE (cm) } & \multicolumn{3}{|c|}{$\mathrm{SDM}(\mathrm{cm})$} & \multicolumn{3}{|c|}{ PSP $(\mathrm{cm})$} & \multicolumn{3}{|c|}{ DFF (day) } \\
\hline & OP & GH & Pooled & OP & GH & Pooled & OP & GH & Pooled & OP & GH & Pooled & OP & GH & Pooled \\
\hline $1 \mathrm{TR}$ & 5.67 & $9.00_{b-g}$ & $7.33_{\mathrm{c}-\mathrm{g}}$ & 62 & $115.00_{\mathrm{a}-\mathrm{c}}$ & $88.50_{\mathrm{c}-\mathrm{h}}$ & $1.36_{j}$ & $1.57_{c-f}$ & $1.47_{\mathrm{k}-\mathrm{o}}$ & 75.56 & $117.67_{a-f}$ & 96.61 & $90.33_{\mathrm{c}-\mathrm{g}}$ & 93 & $91.40_{\mathrm{abc}}$ \\
\hline $2 \mathrm{TL}$ & 5.67 & $7.33_{\mathrm{e}-\mathrm{h}}$ & $6.50_{\text {efg }}^{\circ}$ & 80.1 & $105.17_{b-e}$ & $92.63_{\mathrm{b}-\mathrm{g}}$ & $1.83_{\mathrm{b}-\mathrm{e}}$ & $1.67_{\mathrm{c}-\mathrm{e}}$ & $1.75_{\mathrm{d}-\mathrm{i}}$ & 90 & $97.17_{f-h}$ & 93.58 & $93.00_{a-f}$ & 108.33 & $100.67 \mathrm{a}$ \\
\hline 3 TR & 6.67 & $10.33_{\mathrm{bc}}$ & $8.50_{\mathrm{bcd}}$ & 71.44 & $129.00_{a}$ & $100.22^{\circ}-\mathrm{d}$ & $1.76_{\mathrm{d}-\mathrm{g}}$ & $1.67_{c-e}$ & $1.72_{e-j}$ & 94.78 & $137.50_{a}$ & 116.14 & $94.00_{a-f}$ & 86.33 & $90.17_{a b c}$ \\
\hline 4 TR & 5.5 & $9.33_{b-f}$ & $7.80_{\mathrm{b}-\mathrm{f}}$ & 75.25 & $125.83_{\mathrm{ab}}$ & $105.60_{\mathrm{ab}}$ & $1.53_{\mathrm{g}-j}$ & $1.97_{b}$ & $1.75_{\mathrm{d}-\mathrm{i}}$ & 61 & $125.83_{\mathrm{a}-\mathrm{d}}$ & 99.9 & $94.33_{\mathrm{a}-\mathrm{f}}$ & 95.67 & $95.00_{\mathrm{ab}}$ \\
\hline 5 TR & 8 & $7.67_{\mathrm{d}-\mathrm{h}}$ & $7.80_{\mathrm{b}-\mathrm{f}}$ & 67.11 & $88.00_{\mathrm{de}}$ & $77.56_{\mathrm{hi}}$ & $1.79_{\mathrm{c}-\mathrm{g}}$ & $1.35_{\mathrm{fg}}$ & $1.57_{\mathrm{h}-\mathrm{n}}$ & 86.56 & $98.00_{e-h}$ & 92.28 & $87.00_{f-h}$ & 69.67 & $78.33_{\text {cde }}$ \\
\hline $6 \mathrm{TL}$ & 7 & $11.00_{\mathrm{ab}}$ & $9.00_{\mathrm{abc}}$ & 52.89 & $106.00_{\mathrm{b}-\mathrm{e}}$ & $79.45_{\text {ghi }}$ & $1.42_{\mathrm{h}-\mathrm{j}}^{\circ}$ & $1.55_{\mathrm{d}-\mathrm{g}}$ & $1.49_{\mathrm{k}-\mathrm{o}}$ & 70.11 & $106.00_{c-h}$ & 88.06 & $91.67_{b-g}$ & 86 & $88.83_{\mathrm{abc}}$ \\
\hline $7 \mathrm{TR}$ & 7 & $11.00_{\mathrm{ab}}$ & $9.00_{\mathrm{abc}}$ & 57.67 & $112.67_{a-c}$ & $85.17_{\mathrm{e}-\mathrm{i}}$ & $1.34_{j}$ & $1.64_{\mathrm{c}-\mathrm{e}}$ & $1.49 \mathrm{k}-\mathrm{o}$ & 79.33 & $124.33_{\mathrm{a}-\mathrm{d}}$ & 101.83 & $91.33_{b-\mathrm{g}}$ & 88.33 & $89.83_{\mathrm{abc}}$ \\
\hline $9 \mathrm{ML}$ & 5.67 & $6.50_{\mathrm{h}}$ & $6.00_{\mathrm{fg}}$ & 73.44 & $109.00_{a-d}$ & $87.67_{\mathrm{d}-\mathrm{i}}$ & $1.55_{\mathrm{f}-\mathrm{j}}$ & $1.35_{\mathrm{fg}}$ & $1.45_{1-\mathrm{o}}$ & 74.61 & $128.00_{\mathrm{a}-\mathrm{c}}$ & 95.97 & $96.50_{a-e}$ & 107 & $100.00_{a}$ \\
\hline $10 \mathrm{ML}$ & 3.67 & $9.00_{b-g}$ & $6.33_{\mathrm{efg}}$ & 64.11 & $114.50_{\mathrm{a}-\mathrm{c}}$ & $89.31_{\mathrm{c}-\mathrm{h}}$ & $1.58_{\mathrm{e}-\mathrm{j}}$ & $1.58_{\mathrm{c}-\mathrm{f}}$ & $1.58 \mathrm{~g}-\mathrm{n}$ & 74.22 & $104.83_{\mathrm{d}-\mathrm{h}}$ & 89.53 & $92.00_{\mathrm{a}-\mathrm{g}}$ & 98 & $95.00_{\mathrm{ab}}$ \\
\hline $13 \mathrm{MR}$ & 6.33 & $9.67_{b-e}$ & $8.00_{\mathrm{b}-\mathrm{e}}$ & 70.33 & $106.33_{b-e}$ & $88.33_{\mathrm{c}-\mathrm{h}}$ & $1.84_{b-e}$ & $1.45_{\mathrm{e}-\mathrm{g}}$ & $1.64_{\mathrm{f}-\mathrm{k}}$ & 89.55 & $104.33_{\mathrm{d}-\mathrm{h}}$ & 96.94 & $100.67_{a}$ & 74.33 & $87.50_{a-d}$ \\
\hline $14 \mathrm{ML}$ & 6 & $7.00_{\mathrm{f}-\mathrm{h}}$ & $6.50_{\mathrm{efg}}$ & 63.89 & $96.50_{c-e}$ & $80.20 \mathrm{f}-\mathrm{i}$ & $1.34_{j}$ & $1.29_{\mathrm{g}}^{\mathrm{g}}$ & $1.31_{\mathrm{o}}$ & 77.78 & $87.83_{h}$ & 82.81 & $88.67_{\mathrm{c}-\mathrm{h}}$ & 94 & $91.33_{\mathrm{abc}}$ \\
\hline $15 \mathrm{ML}$ & 8.33 & $10.67_{a-c}$ & $9.50_{\mathrm{ab}}$ & 75.11 & $119.50_{\mathrm{ab}}$ & $97.31_{\mathrm{a}-\mathrm{e}}$ & $2.055_{a-c}$ & $1.76_{\mathrm{b}-\mathrm{d}}^{\mathrm{o}}$ & $1.91_{\mathrm{bcd}}$ & 85.55 & $114.83_{\mathrm{a}-\mathrm{g}}$ & 100.19 & $88.67_{\mathrm{c}-\mathrm{h}}$ & 83.33 & $86.00_{a-e}$ \\
\hline $16 \mathrm{ML}$ & 5 & $10.33_{\mathrm{bc}}$ & $7.67_{\mathrm{b}-\mathrm{f}}$ & 60.66 & $125.33_{\mathrm{ab}}$ & $93.00_{\mathrm{b}-\mathrm{g}}$ & $1.39_{\mathrm{ij}}$ & $1.46_{\mathrm{e}-\mathrm{g}}$ & $1.43 \mathrm{mno}$ & 88.44 & $113.83_{\mathrm{b}-\mathrm{g}}$ & 101.14 & $89.00_{c-h}$ & 87.33 & $88.17_{a-d}$ \\
\hline $17 \mathrm{ML}$ & 6.67 & $9.67_{b-e}$ & $8.17_{\mathrm{b}-\mathrm{e}}$ & 76.22 & $121.17_{a b}$ & $98.69 \mathrm{a}-\mathrm{e}$ & $1.65_{\mathrm{e}-\mathrm{i}}$ & $1.54_{\mathrm{d}-\mathrm{g}}^{\mathrm{g}}$ & $1.60_{\mathrm{f}-\mathrm{m}}$ & 70.11 & $111.83_{\mathrm{b}-\mathrm{g}}$ & 90.97 & $97.33_{a-c}$ & 84 & $92.00_{\mathrm{abc}}$ \\
\hline $18 \mathrm{ML}$ & 6.33 & $9.33_{b-f}$ & $7.83_{\mathrm{b}-\mathrm{f}}$ & 63.39 & $125.50_{\mathrm{ab}}$ & $94.45_{\mathrm{a}-\mathrm{e}}$ & $1.44_{\mathrm{h}-\mathrm{j}}$ & $1.74_{b-d}$ & $1.59_{\mathrm{g}-\mathrm{m}}$ & 66.72 & $115.33_{\mathrm{a}-\mathrm{g}}$ & 91.03 & $97.00_{a-d}$ & 98.33 & $97.67 \mathrm{a}$ \\
\hline $19 \mathrm{ML}$ & 4.67 & $6.67_{\mathrm{gh}}$ & $5.67_{\mathrm{g}}$ & 76.11 & $110.17_{a-c}$ & $93.14_{\mathrm{b}-\mathrm{g}}$ & $1.58_{e-j}$ & $1.33_{\mathrm{fg}}$ & $1.45_{\mathrm{k}-\mathrm{o}}^{\circ}$ & 76.89 & $106.17_{c-h}$ & 91.53 & $91.67_{\mathrm{b}-\mathrm{g}}$ & 91.67 & $91.67_{\mathrm{abc}}$ \\
\hline $21 \mathrm{ML}$ & 4.33 & $9.33_{b-f}$ & $6.83_{\mathrm{d}-\mathrm{g}}$ & 66.89 & $109.50_{a-c}$ & $88.19_{c-h}$ & $2.02_{a-d}$ & $1.65_{c-e}$ & $1.84_{\mathrm{b}-\mathrm{e}}$ & 79.11 & $103.83_{\mathrm{d}-\mathrm{h}}$ & 91.47 & $100.00_{\mathrm{ab}}$ & 100.33 & $100.17_{a}$ \\
\hline $22 \mathrm{ML}$ & 5.33 & $8.33_{c-h}$ & $6.83_{\mathrm{d}-\mathrm{g}}$ & 77.66 & $113.33_{\mathrm{a}-\mathrm{c}}$ & $95.50 \mathrm{a}-\mathrm{e}$ & $2.25 a$ & $1.58_{\mathrm{c}-\mathrm{f}}$ & $1.92_{\mathrm{bcd}}$ & 100.89 & $106.83_{c-h}$ & 103.86 & $88.33_{\mathrm{d}-\mathrm{h}}$ & 88.5 & $88.40_{\mathrm{abc}}$ \\
\hline $23 \mathrm{ML}$ & 7.67 & $10.00_{b-d}$ & $8.83_{a b c}$ & 71.45 & $109.50_{a-c}$ & $90.47_{c-h}$ & $1.67_{\mathrm{e}-\mathrm{h}}$ & $1.85_{\mathrm{bc}}$ & $1.76_{\mathrm{d}-\mathrm{h}}$ & 94.39 & $129.83_{a b}$ & 112.11 & $95.33_{a-f}$ & 90.67 & $93.00_{\mathrm{abc}}$ \\
\hline $25 \mathrm{ML}$ & 7 & $10.00_{b-d}$ & $8.80_{\mathrm{abc}}$ & 72.83 & $97.50_{c-e}$ & $87.63_{\mathrm{d}-\mathrm{i}}$ & $1.83_{\mathrm{b}-\mathrm{e}}$ & $3.83_{a}$ & $2.83_{a}$ & 96.84 & $103.17_{d-h}$ & 100.63 & $90.67_{c-g}$ & 87.67 & $89.17_{a b c}$ \\
\hline $26 \mathrm{CL}$ & 5.5 & $9.67_{b-e}$ & $8.00_{\mathrm{b}-\mathrm{e}}$ & 54.5 & $86.67_{e}$ & $73.80_{i}$ & $1.42_{\mathrm{h}-\mathrm{j}}$ & $1.64_{c-e}$ & $1.53_{\mathrm{j}-\mathrm{n}}$ & 55.84 & $104.67_{d-h}$ & 85.13 & $77.00_{\mathrm{ij}}^{\circ}$ & 80 & $78.50_{\text {cde }}$ \\
\hline $27 \mathrm{CL}$ & 5.67 & $8.67_{b-h}$ & $7.17_{\mathrm{c}-\mathrm{g}}$ & 70.54 & $116.67_{a-c}$ & $93.61_{a-f}$ & $1.45_{\mathrm{h}-\mathrm{j}}$ & $1.66_{c-e}$ & $1.56_{j-n}$ & 70 & $110.17_{b-h}$ & 90.08 & $92.00_{\mathrm{a}-\mathrm{g}}$ & 90 & $91.00_{\mathrm{abc}}$ \\
\hline $29 \mathrm{MN}$ & 6 & $9.00_{b-g}$ & $7.50_{c-g}$ & 73.95 & $129.33_{a}$ & $101.64_{\mathrm{abc}}$ & $2.17 \mathrm{a}$ & $1.82_{b-\mathrm{d}}$ & $1.99_{b}$ & 98.45 & $107.17_{b-h}$ & 102.81 & $87.67_{\text {e-h }}$ & 59 & $73.33_{\mathrm{de}}$ \\
\hline $30 \mathrm{MN}$ & 8 & $13.00_{a}$ & $10.50_{\mathrm{a}}$ & 71.56 & $122.83_{\mathrm{ab}}$ & $97.20_{\mathrm{a}-\mathrm{e}}$ & $1.42_{\mathrm{h}-\mathrm{j}}$ & $1.70_{\mathrm{b}-\mathrm{e}}$ & $1.56_{\mathrm{i}-\mathrm{n}}$ & 85.22 & $109.83_{b-h}$ & 97.53 & $81.00_{\mathrm{hi}}$ & 82.5 & $81.60_{\mathrm{b}-\mathrm{e}}$ \\
\hline $32 \mathrm{MN}$ & 6 & $7.33_{\mathrm{e}-\mathrm{h}}$ & $6.67_{d-g}$ & 85.33 & $129.67 \mathrm{a}$ & $107.50_{a}$ & $1.68_{\mathrm{e}-\mathrm{h}}$ & $1.57_{c-f}$ & $1.63_{\mathrm{f}-1}$ & 94.55 & $105.67_{c-h}$ & 100.11 & $84.00_{\mathrm{g}-\mathrm{i}}$ & 94 & $89.00_{\mathrm{abc}}$ \\
\hline $34 \mathrm{CL}$ & 7 & $6.67_{\mathrm{gh}}$ & $\begin{array}{l}6.80_{\mathrm{d}-\mathrm{g}}\end{array}$ & 86.17 & $112.17_{a-c}$ & $101.77_{\mathrm{abc}}$ & $1.44_{h-j}$ & $1.34_{\mathrm{fg}}$ & 1.39 no & 91.67 & $94.50_{\mathrm{gh}}$ & 93.37 & $69.00_{j}$ & 73.67 & $71.33_{\mathrm{e}}$ \\
\hline $35 \mathrm{CL}$ & 5 & $7.67_{\mathrm{d}-\mathrm{h}}$ & $6.33_{\mathrm{efg}}^{\mathrm{s}}$ & 74.89 & $108.50 \mathrm{a}-\mathrm{d}$ & $91.70_{\mathrm{b}-\mathrm{g}}$ & $1.83_{\mathrm{b}-\mathrm{e}}$ & $1.74_{b-\mathrm{d}}$ & $1.78_{\mathrm{c}-\mathrm{f}}$ & 76.22 & $120.83_{a-e}$ & 98.53 & $70.00_{j}^{\prime}$ & 72.33 & $71.17_{\mathrm{e}}$ \\
\hline Mean & 6.06 & 9.02 & 7.54 & 70.42 & 113.01 & 91.72 & 1.67 & 1.68 & 1.68 & 82.02 & 110.97 & 96.5 & 89.96 & 87.67 & 88.81 \\
\hline $\operatorname{LSD}(p=0.05)$ & n. s & 2.38 & 1.88 & n. s & 21.43 & 13.95 & 0.27 & 0.28 & 0.19 & n. $s$ & 22.98 & n. $s$ & 8.93 & n. $s$ & 14.87 \\
\hline SEM & 0.03 & 0.02 & 0.03 & 0.15 & 0.18 & 0.17 & 0 & 0.01 & 0 & 0.24 & 0.19 & 0.22 & 0.1 & 0.22 & 0.16 \\
\hline
\end{tabular}

Note: OP = open field, GH = glasshouse, NPB = number of primary branches (no.), PHE = plant height 90 days after tra nsplant $(\mathrm{cm}), \mathrm{PSP}=$ plant spread 90 days after tra nsplant $(\mathrm{cm}), \mathrm{LSD}=$ least significant difference, $\mathrm{SEM}=$ standard error of mean, n.s = not significant at $p>0.05$ and mea ns with the same letter in each column also not significantly different at $5 \%$ probability level. 


\subsubsection{Heritability and Genetic Parameters}

Broad-sense heritability, phenotypic coefficient variation, genotypic coefficient variation, and genetic advance are presented in Table 6 . Heritability is a dimension of physical appearance (phenotypic traits) or total variance that is handed down from the parents [25]. We could identify a bigger range of low to high broad-sense heritabilities observed for most of the yield component traits, while low broad-sense heritabilities were evidenced for all vegetative traits. Estimation of broad-sense heritability showed the highest value for the trait fruit girth (FGI) with $77.50 \%$ and the lowest for plant spread (PSP) with 0.00 . Number of fruits per bunch (NFB) and fruit length gave the values of $74.98 \%$ and $63.84 \%$ heritability estimations, respectively. Moderate values (30-60\%) were observed in fruit length to width ratio (FLW) and diameter of fruit (DFR), while the lowest heritability values were illustrated in number of primary branches (NPB), plant height (PHE), stem diameter (SDM), plant spread (PSP), days to first flowering (DFF), total number of fruits (TNF), average fruit weight (AFW), and fruit yield per plant (FYP). In general, for high heritability $(>60 \%)$, values together with high genetic advance $(>20 \%)$ were observed for fruit girth (FGI), fruit length (FLE), and number of fruits per bunch (NFB). These parameters are mainly controlled by the additive type of genes and can be used as selection criteria for significant improvement in fruit yield production of eggplant. The results obtained are in agreement with previous research [30-32]. Nevertheless, moderate heritability values but high genetic advance were observed in diameter of fruit (DFR) and fruit length to width ratio (FLW). Both lower heritability values and genetic advance were respectively indicated by plant height (PHE), stem diameter (SDM), plant spread (PSP), and days to first flowering (DFF). This explanation of the function of non-additive genes in the traits could be corrected by heterosis breeding [33,34].

Next, estimation of the phenotypic coefficient of variance (PCV) and the genotypic coefficient of variance (GCV) ranged from zero to $47.34 \%$, portrayed by plant spread (PSP) and fruit length to width ratio (FLW), respectively. As in overall characteristics of vegetative and yield and their components traits, the highest GCV (>20\%) was evidenced by FGI (30.92\%), FLW (47.34\%), FLE (43.85\%), NFB $(28.72 \%)$, and AFW (30.30\%), while moderate GCV (10-20\%) was observed in NPB (11.69\%), SDM $(10.70 \%)$, DFR $(28.18 \%)$, TNF $(14.97 \%)$, and FYP $(12.69 \%)$. The lowest GCV $(<10 \%)$ was indicated by the remaining PHE $(6.15 \%)$, PSP $(0.00 \%)$, and DFF $(6.92 \%)$, in which their phenotypic expressions were strongly affected by the environment. Hence, the limited selection was found on these traits. Ranges for PCV values from moderate to high were observed as $15.08 \%$ in plant height (PHE) to $80.89 \%$ in the total number of fruit (TNF). High PCV was indicated in TNF $(80.89 \%)$ followed by FYP $(79.04 \%)$, AFW (71.04\%), FLW (61.97\%), FLE (54.89\%), DFR (48.57\%), FGI (35.12\%), NFB (33.69\%), NPB (24.70\%), and SDM (23.18\%), whereas moderate values were recorded for the rest-PHE (15.08\%), PSP $(18.16 \%)$, and DFF (15.59\%). PCV values have undertaken GCV values for all vegetative and yield component traits in correspondence illustrating the prevalence of environmental influence-especially cropping conditions-on traits expressions. This finding is in harmony with previous work of [32,35-39]. Nonetheless, the difference between them proposing the governance of genetics and hence the selection on a phenotypic basis would remain reliable as influenced by environmental factors. This also implicitly shows the importance of germplasm adaptive capacity with the environment used in upcoming crop breeding selection. Higher genotypic coefficient of variation together with high heritability and high genetic advance provide superior indication rather than individual parameters [40]. Fruit girth (FGI), fruit length (FLE), and number of fruits per bunch (NFB) were highlighted trait candidates in meeting these criteria. Thus, it is pivotal to select one trait that gives positive manipulation to the other traits. In addition to the performance response in both vegetative and yield parameters, this study also highlighted the considerably significant degree of genetic variation with the evidence among the accession for some traits that could be further explored for the breeding program. This finding is also in agreement with other researchers, such as [35,41,42]. Additionally, accession selection would be resourceful for an eggplant improvement program established based on yield and yield component traits in particular. 
Table 6. Heritability and genetic variances for quantitative traits.

\begin{tabular}{ccccc}
\hline Traits & $\left.\mathbf{H}_{\mathbf{b}} \mathbf{}_{\mathbf{~}} \mathbf{\%}\right)$ & $\mathbf{G C V} \mathbf{( \% )}$ & $\mathbf{P C V} \mathbf{( \% )}$ & $\mathbf{G A} \mathbf{( \% )}$ \\
\hline NPB & 22.41 & 11.69 & 24.70 & 11.41 \\
PHE & 16.62 & 6.15 & 15.08 & 5.16 \\
SDM & 19.24 & 10.70 & 23.18 & 9.19 \\
PSP & 0.00 & 0.00 & 18.16 & 0.00 \\
DFF & 19.73 & 6.92 & 15.59 & 6.34 \\
FGI & 77.50 & 30.92 & 35.12 & 56.08 \\
DFR & 33.66 & 28.18 & 48.57 & 33.68 \\
FLE & 63.84 & 43.85 & 54.89 & 72.18 \\
FLW & 58.36 & 47.34 & 61.97 & 74.50 \\
TNF & 3.42 & 14.97 & 80.89 & 5.70 \\
NFB & 74.98 & 28.72 & 33.69 & 51.22 \\
AFW & 18.19 & 30.30 & 71.04 & 26.62 \\
FYP & 2.58 & 12.69 & 79.04 & 4.19
\end{tabular}

Note: $\mathrm{H}^{2} \mathrm{~b}(\%)=$ broad se nse heritability, GCV $(\%)=$ genotypic coefficient variance, PCV $(\%)=$ phenotypic coefficient variance, $\mathrm{GA}=$ genetic advance, $\mathrm{NPB}=$ number of primary branches (no.), PHE = plant height 90 days after tra nsplant $(\mathrm{cm}), \mathrm{SDM}=$ stem diameter 90 days after tra nsplant $(\mathrm{cm}), \mathrm{PSP}=$ plant spread 90 days after tra nsplant $(\mathrm{cm}), \mathrm{DFF}=$ days to first flowering (day), FGI = fruit girth $(\mathrm{cm}), \mathrm{DFR}=$ diameter of fruit $(\mathrm{cm}), \mathrm{FLE}=$ fruits length $(\mathrm{cm}), \mathrm{FLW}=$ fruits length to width (ratio), TNF = total number of fruits (no.), NFB = number of fruits per bunch (no.), AFW = average fruits weight (g), $\mathrm{FYP}=$ fruit yield per plant $(\mathrm{g})$

\subsubsection{Cluster Analysis of Agro-Morphological Traits}

Agro-morphological characteristics including vegetative, yield, and yield components parameters were adopted based on the Euclidean distances among the 29 accessions of eggplant to construct a UPGMA dendrogram as in Figure 1. This dendrogram revealed 29 eggplant accessions clustered into six groups with a similarity coefficient of 0.35 and which were the best fit for convenience discussion, and this implied a high level of agro-morphological variation of eggplant accessions. As presented in Table 7, cluster I had four admixed accessions from Thailand and China, namely 1TR, 26CL, 5TR, and 6TL, while cluster II had the largest group of 19 accessions (2TL, 27CL, 20ML, 34CL, 10ML, 22ML, 3TR, 17ML, 18ML, 13MR, 14ML, 16ML, 29MN, 32MN, 7TR, 25ML, 19ML, 15ML, and 30MN). Meanwhile, cluster III had 9ML, 23ML, and 35CL-three admixed accessions from Malaysia and China-and the remaining clusters, IV, V, and VI, had one accession each, 8ML, 21ML, and 4TR, respectively (two accessions from Malaysia and the latter from Thailand). Indeed, there are diverse eggplant accessions commercially domesticated between these three origins, and agro-morphological traits are reliable to classify different accessions in a pool of germplasm [25,43]. Regarding morphological traits' mean performances between clusters, as shown in Table 8, cluster II achieved the best in terms of yield and yield component traits. It portrayed a fruit yield per plant of $1867.95 \mathrm{~g} / \mathrm{plant}$, which was mainly due to the highest total number of fruits (TNF) of 26.47. Utilizing the basis of comparable vegetative and yield components traits, the grouping of individual accessions was exposed by cluster analysis according to similarity and relatedness of eggplants. Hence, the large difference of each accession attribute performance proposed in the crosses between group I and IV or V could be used to attain higher heterosis and vigor among the accessions.

Table 7. List of clusters of 29 eggplant accessio ns according to cluster analysis.

\begin{tabular}{cccc}
\hline Cluster & No. of Accessio ns & Accessio ns & Origin \\
\hline I & 4 & 1TR, 26CL, 5TR, 6TL & Thailand, China \\
II & 19 & 2TL, 27CL, 20ML, 34CL, 10ML, 22ML, 3TR, 17ML, 18ML, 13MR, & Malaysia, Thailand, \\
III & 3 & 14ML, 16ML, 29MN, 32MN, 7TR, 25ML, 19ML, 15ML, 30MN & China \\
IV & 1 & $9 \mathrm{ML}, 23 \mathrm{ML}, 35 \mathrm{CL}$ & Malaysia, China \\
V & 1 & $8 \mathrm{ML}$ & Malaysia \\
VI & 1 & $21 \mathrm{ML}$ & Malaysia \\
\hline
\end{tabular}

Note: accession code abbreviation in Table 1. 


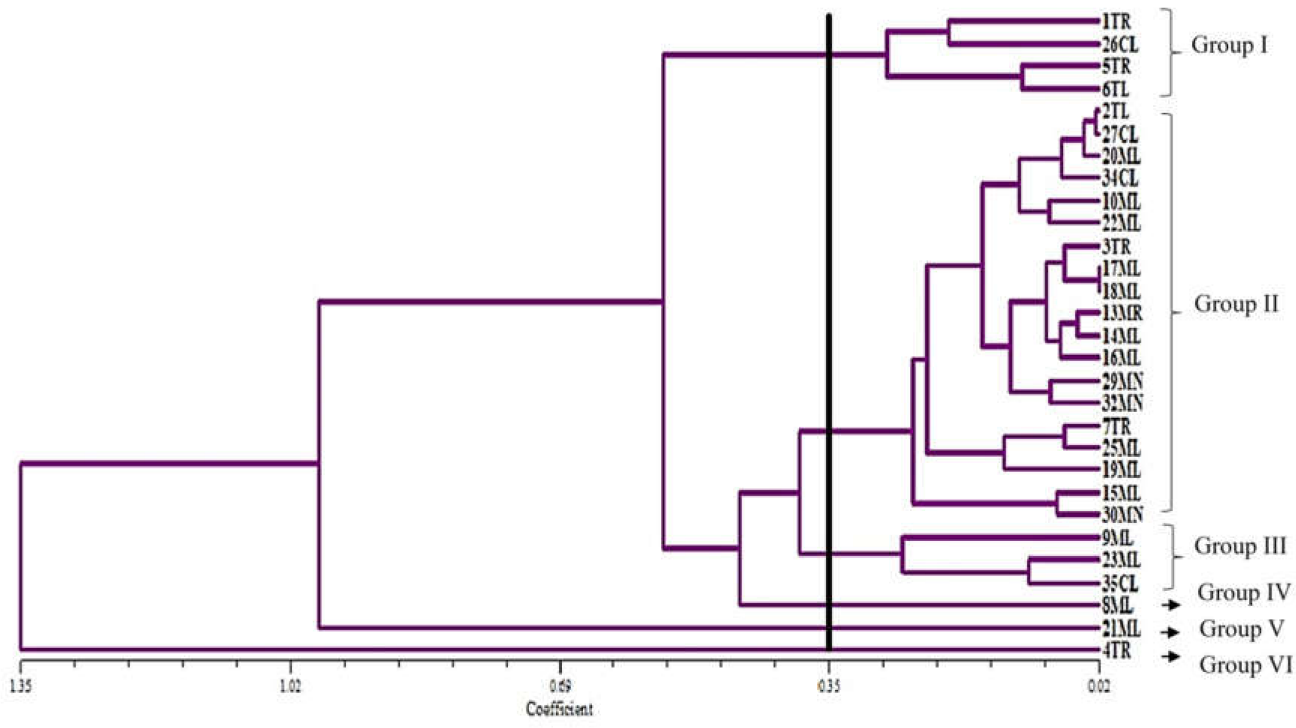

Figure 1. Dendrogram of 29 eggplant accessions based on quantitative traits generated by unweighted pair group method with arithmetic mean (UPGMA) at a 0.35 similarity coefficient.

Table 8. Cluster group and quantitative traits mean.

\begin{tabular}{cccccccccccccc}
\hline CLUSTER & NPB & PHE & SDM & PSP & DFF & FGI & DFR & FLE & FLW & TNF & NFB & AFW & FYP \\
\hline I & 8.03 & 79.83 & 1.52 & 90.52 & 84.27 & 8.34 & 3.32 & 4.64 & 1.79 & 26.37 & 1.52 & 179.98 & 565.50 \\
II & 7.64 & 94.56 & 1.70 & 97.30 & 88.87 & 12.90 & 4.14 & 11.25 & 2.95 & 26.47 & 1.00 & 459.75 & 1867.95 \\
III & 7.05 & 89.95 & 1.66 & 102.20 & 88.06 & 14.92 & 5.72 & 13.53 & 2.74 & 13.94 & 1.00 & 599.07 & 1730.77 \\
IV & 6.67 & 90.42 & 1.76 & 95.58 & 95.83 & 13.34 & 7.28 & 17.12 & 3.34 & 13.50 & 1.00 & 202.80 & 1511.20 \\
V & 6.83 & 88.19 & 1.84 & 91.47 & 100.17 & 20.86 & 6.34 & 8.87 & 1.64 & 15.83 & 1.00 & 962.90 & 1716.90 \\
VI & 7.80 & 105.60 & 1.75 & 99.90 & 95.00 & 16.33 & 5.10 & 6.79 & 1.34 & 4.50 & 1.00 & 165.70 & 323.90 \\
\hline
\end{tabular}

Note: $\mathrm{NPB}=$ number of primary branches (no.), $\mathrm{PHE}=$ plant height 90 days after tra nsplant $(\mathrm{cm}), \mathrm{SDM}=$ stem diameter 90 days after tra nsplant $(\mathrm{cm})$, PSP $=$ plant spread 90 days after tra nsplant $(\mathrm{cm}), \mathrm{DFF}=$ days to first flowering (day), FGI $=$ fruit girth $(\mathrm{cm}), D F R=$ diameter of fruit $(\mathrm{cm}), F L E=$ fruits length $(\mathrm{cm}), F L W=$ fruits length to width (ratio), $\mathrm{TNF}=$ total number of fruits (no.), NFB = number of fruits per bunch (no.), AFW = average fruits weight (g), FYP = fruit yield per plant $(\mathrm{g})$.

\section{Conclusions and Recommendation}

This research revealed that eggplant germplasm had ample genetic variation portrayed through agro-morphological characterization via ANOVA and multivariate analysis. The pivotal understanding of agro-morphological evaluation of genetic variation on eggplant germplasm synergized with cropping condition practices leads to the finding of higher eggplant production with preferable cropping conditions. In Malaysia, it was found that the open field is more suitable for eggplant production with better efficiency of agronomic management together with sustainable production systems. Nevertheless, future work is suggested to explain the molecular approach of genetic variation together with a comprehensive validation of a few seasonal and site trials.

Author Contributions: Conceived and designed the experiments: M.Y.R., J.D., S.I.R., N.N.M.S. and C.P.; Performed the experiment: N.N.M.S. and M.Y.R., Analyzed the data: N.N.M.S., D.R.D. and I.M. Wrote and revised the article: N.N.M.S., M.Y.R., Y.O., J.D., S.I.R. and J.D., Funding acquisition: M.Y.R. and J.D. All authors have read and agreed to the published version of the manuscript.

Funding: This research was funded by the Malaysian Higher Institution Centre of Excellence (HICOE) and Thailand Horticulture Innovation Lab.

Acknowledgments: This project would be impossible without supported Master of Food Security and Climate Change (MSFSCC) project support. Also, the Malaysian Agriculture Research and Development Institute (MARDI) and Research Management Centre (RMC) of Universiti Putra Malaysia (UPM). This may include administrative and technical support of the Institute of Tropical Agriculture and Food Security (ITAFoS), UPM.

Conflicts of Interest: The authors declare no conflict of interest. 


\section{References}

1. Lester, R.N. Origin and domestication of the brinjal eggplant, Solanum melongena, from Solanum incanum, in Africa and Asia. In Solanaceae III: Taxonomy, Chemistry, Evolution; The Linnean Society of London: London, UK, 1991; pp. 369-387.

2. Knapp, S.; Vorontsova, M.S.; Prohens, J. Wild relatives of the eggplant (Solanum melongena L.: Solanaceae): New understanding of species names in a complex group. PLoS ONE 2013, 8, e57039. [CrossRef] [PubMed]

3. FAOSTAT: Agriculture Production Data 2016. Available online: http//www.fao.org/ (accessed on 7 April 2018).

4. DOA (Department of Agriculture Peninsular Malaysia). Vegetables and Cash Crops Statistic, Malaysia. 2018. Available online: http://www.doa.gov.my/index/resources/aktiviti_sumber/sumber_awam/maklumat_ pertanian/perangkaan_tanaman/perangkaan_sayur_tnmn_ladang_2018.pdf (accessed on 21 January 2019).

5. Zainol, A.A.; Izham, A.; Leong, A.C.; Shahadan, M. Production of quality seed and planting materials by MARDI. In Proceedings of the 3rd National Seed Symposium: Prospects and Opportunities, Selangor, Malaysia, 8-9 April 2003; Malaysian Association of Seed Technologies: Selangor, Malaysia, 2003; pp. 32-34.

6. Mahmood, W.J.W. Developing Malaysian seed industry: Prospects and challenges. Econ. Technol. Manag. Rev. 2006, 1, 51-59.

7. Ullah, S.; Ijaz, U.; Shah, T.I.; Najeebullah, M.; Niaz, S. Association and genetic assessment in brinjal. Eur. J. Biotechnol. Biosci. 2014, 2, 41-45.

8. Daunay, M.C.; Lester, R.; Ano, G. Cultivated Eggplants in Tropical Plant Breeding; Charrier, A., Iacquot, M., Hamon, S., Nicolas, D., Enfield, N.H., Eds.; Oxford University Press: Oxford, UK, 2001; pp. 220-225.

9. Causse, M.; Friguet, C.; Coiret, C.; Lépicier, M.; Navez, B.; Lee, M.; Holthuysen, N.; Sinesio, F.; Moneta, E.; Grandillo, S. Consumer preferences for fresh tomato at the European scale: A common segmentation on taste and firmness. J. Food Sci. 2010, 75, S531-S541. [CrossRef]

10. Nwangburuka, C.C.; Kehinde, O.B.; Ojo, D.K.; Denton, O.A.; Popoola, A.R. Morphological classification of genetic diversity in cultivated okra, Abelmoschus esculentus. L Moench using principal component analysis (PCA) and single linkage cluster analysis (SLCA). Afr. J. Biotechnol. 2011, 10, 11165-11172.

11. Oyelola, B.A. The Nigerian Statistical Association Preconference Workshop. In Proceedings of the 2004 Conference Centre, University of Ibadan, Ibadan, Nigeria, 20-21 September 2004.

12. Ulaganathan, V.; Nirmalakumari, A. Finger millet germplasm characterization and evaluation using principal component analysis. SABRAO J. Breed. Genet. 2015, 47, 79-88.

13. Oladosu, Y.; Rafii, M.Y.; Abdullah, N.; Magaji, U.; Miah, G.; Hussin, G.; Ramli, A. Genotype× Environment interaction and stability analyses of yield and yield components of established and mutant rice genotypes tested in multiple locations in Malaysia. Acta Agric. Scand. 2017, 67, 590-606. [CrossRef]

14. Kumar, S.R.; Arumugam, T.; Ulaganathan, V. Genetic diversity in eggplant germplasm by principal component analysis. SABRAO J. Breed. Genet. 2016, 48, 162-171.

15. A'fifah, A.R.; Ismail, M.R.; Puteri, E.M.W.; Abdullah, S.N.A.; Berahim, Z.; Bakhtiar, R.; Kausar, H. Optimum fertigation requirement and crop coefficients of chilli (Capsicum annuum) grown in soilless medium in the tropic climate. Int. J. Agric. Biol. 2015, 17, 80-88.

16. IBPGR. Descriptors for Eggplant; International Board for Plant Genetic Resources: Rome, Italy, 1990.

17. Van der Weerden, G.M.; Barendse, G.W. A web-based searchable database developed for the EGGNET project and applied to the Radboud University Solanaceae database. In Proceedings of the VI International Solanaceae Conference: Genomics Meets Biodiversity, Madison, WI, USA, 23-27 July 2006; pp. 503-506.

18. Rohlf, F.J. NTSYS-Pc: Numerical Taxonomy and Multivariate Analysis System, version 2.1 Exeter Software; Setauket: New York, NY, USA, 2000.

19. Singh, R.K.; Chaudhary, B.D. Biometrical Methods in Quantitative Genetic Analysis; Kalyani Publishers: New Delhi, India, 1979.

20. Sivasubramanian, S.; Madhavamenon, P. Genotypic and phenotypic variability in rice. Madras Agric. J. 1973, 60, 1093-1096.

21. Robinson, H.F.; Comstock, R.E.; Harvey, P.H. Genotypic and phenotypic correlation in corn and their implications in selection. Agron. J. 1949, 43, 282-287. [CrossRef]

22. Assefa, K.; Ketema, S.; Tefera, H.; Nguyen, H.T.; Blum, A.; Ayele, M.; Bai, G.; Simane, B.; Kefyalew, T. Diversity among germplasm lines of the Ethiopian cereal tef [Eragrostis tef (Zucc.) Trotter]. Euphytica 1999, 106, 87-97. [CrossRef] 
23. Johnson, H.W.; Robinson, H.F.; Cormstock, R.E. Estimation of genetic and environmental variability in soybeans. Agron. J. 1955, 47, 314-318. [CrossRef]

24. Juangsamoot, J.; Ruangviriyachai, C.; Techawongstien, S.; Chanthai, S. Determination of capsaicin and dihydrocapsaicin in some hot chillies varieties by RP-HPLC-PDA after magnetic stirring extraction and clean up with C18 cartridge. Int. Food Res. J. 2012, 19, 1217-1226.

25. Oladosu, Y.; Rafii, M.Y.; Abdullah, N.; Abdul Malek, M.; Rahim, H.A.; Hussin, G.; Kareem, I. Genetic variability and selection criteria in rice mutant lines as revealed by quantitative traits. Sci. World J. 2014, 2014. [CrossRef]

26. Caguiat, X.G.I.; Hautea, D.M. Genetic diversity analysis of eggplant (Solanum melongena. L.) and related wild species in the Philippines using morphological and SSR markers. SABRAO J. Breed. Genet. 2014, 46, 183-201.

27. Naujeer, H.B. Morphological Diversity in Eggplant (Solanum melongena L.), Their Related Species and Wild Types Conserved at the National Gene Bank in Mauritius. Master's Thesis, Swedish University of Agriculture Sciences, Uppsala, Sweden, 2009.

28. Katsoulas, N.; Stanghellini, C. Modelling crop transpiration in greenhouses: Different models for different applications. Agronomy 2019, 9, 392. [CrossRef]

29. Xiao, N.; Pan, L.L.; Zhang, C.R.; Shan, H.W.; Liu, S.S. Differential tolerance capacity to unfavourable low and high temperatures between two invasive whiteflies. Sci. Rep. 2016, 6, 1-10. [CrossRef]

30. Pathania, N.K.; Rajeev Katoch, D.R.; Chaudhary, C.K.S. Genetic variability and association studies in eggplant. In Proceedings of the International Conference on Vegetables 2002, Bangalore, India, 11-14 November 2002; p. 89.

31. Chung-Won Bok, J.S.; Oh-Jusung, H.W.; Chung, W.B.; Jeong, P.S. Hwang (Genetic analysis of $F_{1}$ generation in eggplant. J. Korean Soc. Hort. Sci. 2003, 44, 44-48.

32. Kumar, S.R.; Arumugam, T.; An, C.R.; Premalakshmi, V. Genetic variability for quantitative and qualitative characters in Brinjal (Solanum melongena L.). Afr. J. Agric. Res. 2013, 8, 4956-4959.

33. Ridzuan, R.; Rafii, M.Y.; Mohammad Yusoff, M.; Ismail, S.I.; Miah, G.; Usman, M. Genetic diversity analysis of selected Capsicum annuиm 1. Genotypes based on morphophysiological, yield characteristics and their biochemical properties. J. Sci. Food Agric. 2019, 99, 269-280. [CrossRef]

34. Priyanka, B.; Naidu, M. Genetic variability, heritability and genetic advance of growth and yield components of chilli (Capsicum annuum L.) genotypes. Int. J. Sci. Res. 2016, 5, 1305-1307.

35. Akpan, N.M.; Ogbonna, P.E.; Onyia, V.N.; Okechukwu, E.C.; Atugwu, I.A. Variability studies on ten genotypes of eggplant for growth and yield performance in south eastern Nigeria. J. Anim. Plant Sci. 2016, $26,1034-1041$.

36. Mohammad, R.N.R.; Mahdiyeh, P.; Abdolrahim, G.; Javad, A. Variability, heritability and association analysis in eggplant (Solanum melongena L.). ARPN J. Agric. Biol. Sci. 2015, 10, 464-468.

37. Mili, C.; Bora, G.C.; Das, B.J.; Paul, S.K. Studies on variability, heritability and genetic advance in Solanum melongena 1. (brinjal) genotypes. Direct Res. J. Agric. Food Sci. 2014, 2, $192-194$.

38. Babu, S.; Patil, R. Genetic variability and correlation studies in eggplant (Solanum melongena L.). Madras J. Aric Res. 2005, 95, 18-23.

39. Singh, O.; Kumar, J. Variability, heritability and genetic advance in brinjal. Indian J. Hort. 2005, 62, $265-267$.

40. Shabanimofrad, M.; Rafii, M.Y.; Wahab, P.M.; Biabani, A.R.; Latif, M.A. Phenotypic, genotypic and genetic divergence found in 48 newly collected Malaysian accessions of Jatropha curcas L. Ind. Crop. Prod. 2013, 42, 543-551. [CrossRef]

41. Senapati, N.; Mishra, H.N.; Bhoi, M.K.; Dash, S.K.; Prasad, G. Genetic variability and divergence studies in brinjal (Solanum melongena L.). Veg. Sci. 2009, 36, 150-154.

42. Kushwah, S.; Bandyopadhyay, B.B. Variability and correlation studies in brinjal. Indian J. Hortic. 2005, 62, 210-212.

43. Franco, J.; Crossa, J.; Ribaut, J.M.; Bertran, J.; Warburton, M.L.; Khairallah, M. A method for combining molecular markers and phenotypic attributes for classifying plant genotypes. Theor. Appl. Genet. 2001, 103, 944-952. [CrossRef]

(C) 2020 by the authors. Licensee MDPI, Basel, Switzerland. This article is an open access article distributed under the terms and conditions of the Creative Commons Attribution (CC BY) license (http://creativecommons.org/licenses/by/4.0/). 